\title{
Sensitivity Analysis and Probabilistic Re-entry Modeling for Debris using High Dimensional Model Representation based Uncertainty Treatment
}

\author{
Piyush M. Mehta ${ }^{1, *}$, Martin Kubicek ${ }^{2}$, Edmondo Minisci $^{3}$, and \\ Massimiliano Vasile ${ }^{4}$
}

\section{Abstract}

Well-known tools developed for satellite and debris re-entry perform breakup and trajectory simulations in a deterministic sense and do not perform any uncertainty treatment. The treatment of uncertainties associated with the re-entry of a space object requires a probabilistic approach. A Monte Carlo campaign is the intuitive approach to performing a probabilistic analysis, however, it is computationally very expensive. In this work, we use a recently developed approach based on a new derivation of the high dimensional model representation method for implementing a computationally efficient probabilistic analysis approach for re-entry. Both aleatoric and epistemic uncertainties that affect aerodynamic trajectory and ground impact location are considered. The method is applicable to both controlled and un-controlled re-entry scenarios. The resulting ground impact distributions are far from the typically used Gaussian or ellipsoid distributions.

Keywords: Debris; Re-entry; Ground-Impact; Probabilistic-Distribution; Modeling

\section{*Corresponding Author}

Email address: piyushmukeshmehta@gmail.com (Piyush M. Mehta)

${ }^{1}$ Marie Curie Fellow, Department of Mechanical and Aerospace Engineering, University of Strathclyde, Glasgow UK

${ }^{2} \mathrm{PhD}$ Candidate, Department of Mechanical and Aerospace Engineering, University of Strathclyde, Glasgow UK

${ }^{3}$ Lecturer, Department of Mechanical and Aerospace Engineering, University of Strathclyde, Glasgow UK

${ }^{4}$ Professor, Department of Mechanical and Aerospace Engineering, University of Strathclyde, Glasgow UK 


\section{Introduction}

Space Situational Awareness (SSA) is quickly becoming imperative for nations around the world, especially those with space capabilities and assets. As the low Earth orbit (LEO) debris and spacecraft population that have exceeded their operational lifetime rises each year, the rate at which objects re-enter the Earth's atmosphere will also steadily rise. Most of these objects will probably not reach the ground for impact; however, parts of large objects like rocket bodies and satellites or resident space objects (RSOs) with mass greater than a ton have a high probability of surviving the harsh re-entry environment. The surviving parts can be hazardous (e.g. fuel tanks with unused hydrazine or radioactive components) and can cause damage and casualties within a populated area.

The National Aeronautics and Space Administration [NASA-STD-8719.14. (2012)] and European Space Agency [ESA (2008)] guidelines require that any RSO re-entering the atmosphere (1) fully demise in the atmosphere, or (2) impact with an energy of no more than 15 Joules with an associated casualty risk of less than 1 in 10000. Complying with these guidelines require an end-of-life analysis for all future planned missions. The impact location of an object re-entering the atmosphere is affected by uncertainties in initial conditions, atmospheric characteristics, and object properties, as well as break-up/fragmentation events. Therefore, it is important to have an accurate estimate of not just the deterministic impact location but also the statistical distribution due to the uncertainties involved. Existing re-entry modeling tools used by space agencies are either deterministic, proprietary, non-open source, and/or are not freely available to the research community [Rochelle et al. (1999), Koppenwallner et al. (2005), Martin et al. (2005)].

All the existing tools have five basic building blocks: i) Aerodynamics, ii) Aerothermodynamics, iii) Flight Dynamics, iv) Structural analysis, and v) Thermal analysis, and can be classified as either spacecraft- or objectoriented. Spacecraft-oriented tools perform the analysis with higher fidelity compared to object-oriented tools (for e.g. 6DoF vs 3DoF dynamics or detailed thermal analysis vs lumped mass approach), however they are harder to use and computationally expensive because spacecraft-oriented tools constantly share data between the building blocks whereas object-oriented tools use the blocks independently. Therefore, a logical approach would ideally 
involve using an object-oriented tool for the preliminary analysis, followed by a more concentrated campaign with a spacecraft-oriented tool based on the preliminary results from the object-oriented tool. The only spacecraftoriented tool known to exist is SCARAB (Spacecraft Atmospheric Re-entry and Aero-thermal Break-up) developed by HTG under a contract from ESA [Koppenwallner et al. (2005)].

The object-oriented approach uses a simplified representation of the reentering object made up of primitive shapes such as sphere, cylinder, cone, etc. The approach assumes or calculates a demise altitude following which the object is assumed to break-up into multiple objects, represented by the individual primitive geometries [Rochelle et al. (1999), Martin et al. (2005), and Parigini et al. (2015)]. Figure 1 shows the idea behind an object-oriented tool.

As previously mentioned, an accurate end-of-life analysis involves a probabilistic approach due to the uncertainties involved. A Monte Carlo (MC) campaign is the most intuitive approach available for uncertainty quantification and propagation. Figure 2 shows an example of a MC campaign run using Deimos' proprietary object-oriented tool DEBRIS [Parigini et al. (2015)]. The trajectories in blue show a limiting case representative of shallow un-controlled re-entry, cyan represents a 'normal' controlled re-entry while the orange color represents a limiting case of controlled re-entry at a highly steep flight path angle (not realistic). DEBRIS is one of a very few, if not the only, new-age re-entry tool that performs uncertainty-treatment albeit expensively.

The MC approach can provide a realistic distribution for the output of interest $(F(\mathbf{x}))$ due to the uncertainties in the input parameters, however, it does so with a very high computational cost. Moreover, a sensitivity study that can provide qualitative and quantitative insights into the effects of uncertainties in input parameters on the output and influence engineering design decisions is typically not feasible. Even though MC based methods are among the most popular used for sensitivity studies, they require a large amount of sampling of the stochastic input domains and expensive function evaluations to estimate the statistical properties of the given model. The model code is typically assumed to be a black-box and will henceforth be refereed to as the black-box model (BBM) in this paper.

The well known Sobol sensitivity method [Saltelli et al. (2004), Saltelli et al. (2008)] is also based on the MC approach and decomposes the variance of $F(\mathbf{x})$ for the given BBM into parts attributable to input variables. Let's 
assume we have $n$ uncertain input parameters; a sensitivity study accounting for only first order effects would require $n+1 \mathrm{MC}$ campaigns, each coming at a very high cost. The outcome of the method is the quantification of the influence of each variable in the given BBM. Scatter plots can be used to gain insights into the BBM, visualize the influence of a variable, and estimate the behavior of $F(\mathbf{x})$ in a given domain [Kleijnen and Helton (1999a), Kleijnen and Helton (1999b), Saltelli et al. (2004), Saltelli et al. (2008)], however, they require a significant amount of experience in sensitivity analysis.

The computational cost of performing a sensitivity analysis can be significantly reduced by building a cheap surrogate model for the BBM. Several different methods exist for surrogate development such as the Stochastic Collocation (SC) method [Eldred and Burkardt (2009a), Eldred (2009b)], the Polynomial Chaos (PC) method [Wiener (1938), Hosder et al. (2006), Eldred et al. (2008), Eldred and Burkardt (2009a), Eldred (2009b), Cheng et al. (2010), Togawa et al. (2011), Branicki and Majda (2013)], the Kriging surrogate model method [Fang et al. (2006), Lee and Kwon (2008), Forrester et al. (2008)], and the Pade-Legendre approximation method. These methods are non-intrusive by nature, i.e. as previously mentioned, they consider the model code of interest as a black box. In the work of Sudret [Sudret (2008)], the non-intrusive PC is coupled with the Sobol method for directly computing the Sobol Indices from the PC expansion.

In the case of other non-intrusive methods, the sensitivity indices are obtained by sampling the cheap surrogate models via the MC approach. Unfortunately, one of the biggest limitations of surrogate modeling approach is the so called Curse of Dimensionality (CoD), introduced by Richard Bellman [Bellman (1961)], which limits the use of surrogate based approaches to functions with a small number of independent parameters, and the use of non-intrusive methods to problems with a small number of stochastic input dimensions. Various sampling techniques have been proposed to mitigate the CoD problem. The Latin Hyper-cube sampling (LHS) [Fang et al. (2006)] has been successfully used for certain problems [Mehta et al. (2014)] with other approaches such as LaPSO [Chen et al. (2013)], Uniform Design (UD) [Fang et al. (2006)] or Hammersley Sampling [Walters et al. (2007), Cheng et al. (2010)] also available. In the framework of Uncertainty Quantification (UQ) problems, Smolyak Sparse grid [Gerstner and Gribel (1998), Barthelmann et al. (2000), pflueger (2010)] and its different variations have become popular techniques, which combined with Non-intrusive Polynomial Chaos (NIPC) gives very accurate results for a low number of samples. Unfortu- 
nately, even this approach is not affordable because of high cardinality [Gaoa and Hesthavenb (2010)].

In this work, uncertainty treatment is performed using a novel highdimensional derivative based uncertainty quantification and propagation (UQ\&P) approach with an inherent sensitivity analysis study developed recently at the University of Strathclyde [Kubicek et al. (2015)]. The approach is based on cut-HDMR (High Dimensional Model Representation) and multi-surrogate adaptive sampling where the surrogate model development is followed by a sensitivity analysis in an iterative sense addressing all the problems previously discussed. The approach is able to highlight some important aspects of a given problem (atmospheric re-entry in this instance) by decomposing the stochastic space into sub-domains, which are then interpolated separately. Each sub-domain is independent and provides insight into the influence of each stochastic input variable on $F(\mathbf{x})$, where the outcome on each subdomain can be visualized with histograms. The histograms represent the output from direct sampling of the surrogates for each sub-domain.

The $F(\mathbf{x})$ 's in the current study are the longitude and latitude of the ground impact location, while the uncertain input parameters considered are atmospheric properties (density, temperature, composition, and free-stream air heat capacity), initial conditions (re-entry flight path angle, speed, and direction angle) as well as the mass of the object. The study is performed with a spherical object undergoing a planar re-entry. Initial conditions representative of a re-entry from a circular orbit are used under only the effects of gravity and drag. The HDMR UQ\&P method is applicable to both controlled and un-controlled re-entry scenarios and is validated using three different simulations representative of : i) un-controlled 'shallow' re-entry (flight path angle - $\gamma \approx 0$ degrees), ii) controlled 'normal' re-entry ( $\gamma \approx-1.25$ degrees), and iii) controlled 'steep' re-entry ( $\gamma \approx-5$ degrees). The 'shallow' and 'normal' $\gamma$ 's are derived from the Automated Transfer Vehicle (ATV) missions whereas $\gamma$ for the 'steep' re-entry is an assumed realistic limiting value. The results are validated by comparison with MC simulations directly using the BBM.

The work is part of a development effort for a Free Open Source Tool for Re-entry of Asteroids and Space Debris (FOSTRAD) to overcome the nonfreely available, non-open source, and/or proprietary state of the existing tools. The goal with FOSTRAD is to have higher fidelity than object-oriented tools while avoiding the complexity of a spacecraft-oriented approach. The framework for FOSTRAD is modular in nature where work on modules for 
atmospheric entry of asteroids or near Earth objects [Mehta et al., (2015a), Mehta et al. (2016a)], and improved aerodynamic and aerothermodynamic models for simple primitive and complex geometries [Mehta et al. (2015b), Mehta et al. (2016b)] has been recently performed. The current work feeds into the UQ\&P module of FOSTRAD.

The paper is organized in the following format: the next section discusses the methodology including trajectory dynamics and aerodynamics, and the high-dimensional UQ\&P approach, followed by a section that describes the simulation cases with presentation and discussion of the results. The last section provides a summary and draws conclusion on the present work and provides recommendations for future work and direction.

\section{Methodology}

\subsection{Trajectory Dynamics}

The trajectory dynamics and aerodynamic models make up the BBM for the current study. The atmospheric entry simulation is set to begin at an altitude of $120 \mathrm{~km}$. A simple spherical object re-entering the Earth's atmosphere is modeled as a point mass and tracked through the atmosphere down to ground. The dynamics of the object is governed by the following system of differential equations:

$$
\begin{gathered}
\dot{h}=V_{\infty} \sin \gamma \\
\dot{V}_{\infty}=-\frac{D}{m}-g \sin \gamma+\omega_{E}^{2}\left(R_{E}+h\right) \cos \phi(\sin \gamma \cos \phi-\cos \gamma \cos \chi \sin \phi) \\
\dot{\gamma}=\left(\frac{V_{\infty}}{R_{E}+h}-\frac{g}{V_{\infty}}\right) \cos \gamma+2 \omega_{E} \sin \chi \cos \phi+ \\
+\omega_{E}^{2}\left(\frac{R_{E}+h}{V_{\infty}}\right) \cos \phi(\cos \chi \sin \gamma \sin \phi+\cos \gamma \cos \phi) \\
\dot{\chi}=-\left(\frac{V_{\infty}}{R_{E}+h}\right) \cos \phi \sin \chi \tan \phi+2 \omega_{E}(\sin \phi-\cos \chi \cos \phi \tan \gamma)- \\
-\omega_{E}^{2}\left(\frac{R_{E}+h}{V_{\infty} \cos \gamma}\right) \cos \phi \sin \gamma \sin \chi
\end{gathered}
$$




$$
\begin{aligned}
& \dot{\phi}=\left(\frac{V_{\infty}}{R_{E}+h}\right) \cos \gamma \cos \chi \\
& \dot{\lambda}=\left(\frac{V_{\infty}}{R_{E}+h}\right) \frac{\cos \gamma \sin \chi}{\cos \phi}
\end{aligned}
$$

where $h$ is the altitude, $V_{\infty}$ is the speed of the object, $\gamma$ is the flight path angle, $D$ is the drag force, $g$ is the gravitational acceleration, $\omega_{E}$ is the Earth's rotational speed, $R_{E}$ is the radius of the Earth, $\chi$ is path direction angle, and $\phi$ and $\lambda$ are latitude and longitude, respectively. The gravitational acceleration is modeled as a function of the altitude given as:

$$
g(h)=g_{0}\left(\frac{h}{R_{E}+h}\right)^{2}
$$

where the value of $g_{0}$ is $9.81 \mathrm{~ms}^{-2}$.

\subsection{Aerodynamics}

The re-entering object is modeled with a mesh comprising of triangular facets. The pressure and shear contribution of each facet (visible to the flow) to aerodynamics is computed independently as a function of the local flow inclination angle, $\theta$. The axial and normal force coefficients are computed with integrals of the pressure and shear distributions over the surface.

\subsubsection{Continuum Flow Regime}

The aerodynamic contribution in the continuum flow regime is computed using the Modified Newtonian Theory (MNT) given as [Newton (1946)]:

$$
C_{p}=C_{p, \max } \sin ^{2} \theta
$$

where $C_{p}$ is the local pressure coefficient and $C_{p, \max }$ is the maximum or stagnation point pressure coefficient. The shear contribution in the continuum regime is assumed to be zero. 


\subsubsection{Free Molecular Flow Regime}

The aerodynamic contribution of each facet in the free molecular (FM) regime is computed using Schaaf and Chambre's closed-form analytic model given in Eqs. 9 and 10 that accounts for both pressure and shear [Schaaf and Chambre (1958)].

$$
\begin{aligned}
& C_{p}=\frac{1}{s^{2}} {\left[\left(\frac{2-\sigma_{N}}{\sqrt{\pi}} s \sin \theta+\frac{\sigma_{N}}{2} \sqrt{\frac{T_{w}}{T_{\infty}}}\right) e^{-(s \sin \theta)^{2}}+\right.} \\
&\left.+\left\{\left(2-\sigma_{N}\right)\left((s \sin \theta)^{2}+\frac{1}{2}\right)+\frac{\sigma_{N}}{2} \sqrt{\frac{\pi T_{w}}{T_{\infty}}} s \sin \theta\right\}(1+\operatorname{erf}(s \sin \theta))\right]
\end{aligned}
$$

$C_{\tau}=-\frac{\sigma_{T} \cos \theta}{s \sqrt{\pi}}\left[e^{-(s \sin \theta)^{2}}+\sqrt{\pi} s \sin \theta(1+\operatorname{erf}(s \sin \theta)]\right.$

where $C_{p}$ and $C_{\tau}$ are the pressure and shear coefficients, respectively, $\sigma_{N}$ and $\sigma_{T}$ are the normal and tangential momentum accommodation coefficients, respectively, $T_{w}$ is the surface or body wall temperature, $T_{\infty}$ is the free stream translational temperature, $V_{\infty}$ is the object or free stream velocity, $\operatorname{erf}()$ is the error function, and $s$ is the speed ratio given as:

$$
s=\frac{V_{\infty}}{\sqrt{2 R T_{\infty}}}
$$

where $R$ is the universal gas constant.

\subsubsection{Transition Flow Regime}

Aerodynamic computations in the transition regime are performed using the recently developed sigmoid bridging functions [Mehta et al. (2015c), Mehta et al. (2015b)]. The developed function uses the sigmoid (base 10) as the basis function. Optimized accuracy and complexity is achieved using two sigmoid functions as given in Eq.(12)

$$
\begin{aligned}
C_{X_{\text {trans }}}=C_{X_{c}}+\left(C_{X_{f m}}-C_{X_{c}}\right) & {\left[a_{s i} \operatorname{sig}_{10}\left(b_{s i} \log _{10}(K n)+c_{s i}\right)+\right.} \\
& \left.+d_{s i} \operatorname{sig}_{10}\left(e_{s i} \log _{10}(K n)+f_{s i}\right)+g_{s i}\right]
\end{aligned}
$$


where $(a-g)_{s i}$ are fitting constants and

$$
\operatorname{sig}_{10}=\frac{1}{1+10^{(-/+) x}}
$$

where the sign of the exponent depends on the trend of the coefficient across the transition region.

\subsection{Uncertainty Treatment}

\subsubsection{High Dimensional Model Representation}

This work uses the recently developed high dimensional uncertainty propagator based on a cut-HDMR decomposition approach. If $F(\mathbf{x})$ is a derivable and integrable function defined on a $n$-dimensional ( $n$ being the number of random input variables) unit hypercube - $[0,1]^{n}$ and $\mathbf{x} \in[0,1]^{n}$, the ANOVA representation of $F(\mathbf{x})$ can be given as:

$$
F(\mathbf{x})=F_{0}+\sum_{i=1}^{n} F_{i}\left(x_{i}\right)+\sum_{1 \leq i<j \leq n} F_{i, j}\left(x_{i}, x_{j}\right)+\ldots+F_{1, \ldots, n}\left(x_{1}, \ldots, x_{n}\right)
$$

where $F_{0}$ is the constant term and represents the mean value of $F(\mathbf{x}), F_{i}\left(x_{i}\right)$ represents the contribution of the variable $x_{i}$ to $F(\mathbf{x}), F_{i, j}\left(x_{i}, x_{j}\right)$ represents the pair correlated contribution to $F(\mathbf{x})$ of the input variables $x_{i}$ and $x_{j}$, $1 \leq i<j \leq n$, etc. Each term in the Eq. (14) is independent and represents the contribution to the final function. The last term $F_{1, \ldots, n}\left(x_{1}, \ldots, x_{n}\right)$ contains the correlated contribution of all input variables and the total number of summands for Eq. (14) is $2^{n}$.

Each independently differentiable and integrable term in Eq. (14) is differentiated according to its generic variables to obtain the infinitesimal increment in $F(\mathbf{x})$, leading to the following equation:

$$
d F(\mathbf{x})=\sum_{i=1}^{n} \frac{\partial F(\mathbf{x})}{\partial x_{i}} d x_{i}+\sum_{1 \leq i<j \leq n} \frac{\partial F(\mathbf{x})}{\partial x_{i}, x_{j}} d x_{i} d x_{j}+\ldots+\frac{\partial F(\mathbf{x})}{\partial x_{1}, \ldots, x_{n}} d x_{1} \ldots d x_{n}
$$

Equation (15) relates an infinitesimal change in $F(\mathbf{x})$ due to an infinitesimal change in the stochastic input variables. Eq. (15) may resemble the Taylor series expansion, but it is fundamentally different. The Taylor expansion considers the coefficients of each derivative and also higher order derivatives along one direction (e.g. $\left.\frac{\partial F(\mathbf{x})}{\partial x_{1} \partial x_{1}} \ldots \frac{\partial F(\mathbf{x})}{\partial x_{n} \partial x_{n}}\right)$, whereas the ANOVA 
representation in Eq. (15) does not consider derivative coefficients and only one derivative in each direction. The differential equation as represented in Eq. (15) is impractical, therefore, an integral form of Eq. (15) is introduced as

$$
\begin{aligned}
F(\mathbf{x})-F\left({ }^{\mathbf{c}} \mathbf{x}\right)=\sum_{i=1}^{n} \int_{{ }^{x_{i}}}^{x_{i}} \frac{\partial F(\xi)}{\partial \xi_{i}} d \xi_{i}+ & \sum_{1 \leq i<j \leq n} \int_{{ }^{x_{x_{i}}}}^{x_{i}} \int_{{ }^{x_{x_{j}}}}^{x_{j}} \frac{\partial F(\xi)}{\partial \xi_{i}, \xi_{j}} d \xi_{i} d \xi_{j}+\ldots \\
& +\int_{{ }_{x_{1}}}^{x_{1}} \ldots \int_{{ }^{x_{n}}}^{x_{n}} \frac{\partial F(\xi)}{\partial \xi_{1}, \ldots, \xi_{n}} d \xi_{1} \ldots d \xi_{n}
\end{aligned}
$$

where ${ }^{\mathbf{c}} \mathbf{x}$ represents the central position in the stochastic space called the central point and, generally, considered to be the statistical mean of a given stochastic input variable. The terms in Eq. 16 representing the integral of the derivative of each independent function are defined as an increment function. Each increment function provides an independent contribution to the final solution and therefore can be modeled using an independent surrogate. This use of multiple independent surrogates represents the major difference between the method used in this work and the currently used uncertainty propagation methods. The current method is described in detail in the work of [Kubicek et al. (2015)].

The non-important stochastic spaces/interactions are neglected in order to decrease the number of expensive BBM calls. The stochastic space reduction is done in two steps: the first evaluates the importance of the increment functions, and the second neglects the zeroth increment functions. The evaluation of the importance is based on fundamental aspects of the integral form and inverse logic. The importance of each interaction is evaluated and its contribution is added to the final model. The process is stopped when the desired accuracy is achieved. The neglect step, as the name suggests, neglects all zeroth order increment functions that are passed through the evaluation phase. After selecting the important increment functions, the method switches to an automatic sampling approach and interpolates each increment function separately leading to an optimal number of BBM calls.

The multi-dimensional Lagrange surrogate model is used in this work; however, different techniques can be used due to the modular nature of the method. The adaptive sampling process takes into account the behavior of the increment function and the probability distribution of the stochastic input variable. The behavior is captured using the Error Comparison (EC) 
function that is later modified to take into account the probability distribution of the stochastic input variable. The convergence criterion is based on the observation of the standard deviation and the expected value. The statistical properties are obtained using MC sampling on the surrogate model for each increment function. This allows the visualization of each increment function separately (i.e. the probability distribution function (PDF) for each stochastic input variable.) as well as the final PDF.

\subsubsection{Sensitivity Analysis}

The sensitivity of each increment function represents the influence of the corresponding stochastic input variable (or combination of variables) on $F(\mathbf{x})$. The orthogonal property of the increment functions allows for each function to be handled separately i.e. each increment function statistics is calculated independently. This allows to define the statistical properties of the first order increment function as follows:

$$
\begin{aligned}
\mu_{i} & =\int_{-\infty}^{\infty} \int_{c_{x_{i}}}^{x_{i}} \frac{\partial F(\xi)}{\partial \xi_{i}} d \xi_{i} p_{i}\left(x_{i}\right) d x_{i} \\
\sigma_{i}^{2} & =\int_{-\infty}^{\infty}\left(\int_{{ }_{x_{i}}}^{x_{i}} \frac{\partial F(\xi)}{\partial \xi_{i}} d \xi_{i}-\mu_{i}\right)^{2} p_{i}\left(x_{i}\right) d x_{i}
\end{aligned}
$$

which can be extended to higher order increment increment function, i.e. interaction terms, in the following way

$$
\begin{aligned}
\mu_{k} & =\int_{-\infty}^{\infty} \ldots \int_{-\infty}^{\infty} d F_{k} p_{k}(\mathbf{x}) d x_{i} \ldots d x_{j} \\
\sigma_{k}^{2} & =\int_{-\infty}^{\infty} \ldots \int_{-\infty}^{\infty}\left(d F_{k}-\mu_{k}\right)^{2} p_{k}(\mathbf{x}) d x_{i} \ldots d x_{j}
\end{aligned}
$$

where $d F_{k}$ represents the increment function defined in Eq. 16, i.e.

$$
d F_{k}=\int_{{ }^{c} x_{i}}^{x_{i}} \ldots \int_{{ }^{x_{j}}}^{x_{j}} \frac{\partial F(\xi)}{\partial \xi_{i} \ldots \xi_{j}} d \xi_{i} \ldots d \xi_{j}
$$

and $\mu_{k}$ with $k=n+1, \ldots, 2^{n}-1$ represents the partial mean and $\sigma_{k}^{2}$ represents the partial variance. The mean and variance sensitivity indices for each increment function are defined as:

$$
S_{i / k}^{\sigma}=\frac{\sigma_{i / k}^{2}}{\sigma^{2}}
$$




$$
S_{i / k}^{\mu}=\frac{\mu_{i / k}}{\mu}
$$

where $i$ represents the first order increment functions, $k$ represents the higher order increment functions, and where $\sigma^{2}$ and $\mu$ are computed as follows:

$$
\begin{aligned}
\sigma^{2}=\int_{-\infty}^{\infty}(F(\mathbf{x})-\mu)^{2} p(\mathbf{x}) d \mathbf{x}= & \int_{-\infty}^{\infty} \ldots \int_{-\infty}^{\infty}\left(\left(F\left({ }^{\mathbf{c}} \mathbf{x}\right)+\sum_{i=1}^{n} \int_{{ }^{x_{i}}}^{x_{i}} \frac{\partial F(\xi)}{\partial \xi_{i}} d \xi_{i}+\right.\right. \\
& +\sum_{1 \leq i<j \leq n} \int_{{ }^{x_{x_{i}}}}^{x_{i}} \int_{{ }_{c_{x_{j}}}}^{x_{j}} \frac{\partial F(\xi)}{\partial \xi_{i}, \xi_{j}} d \xi_{i} d \xi_{j}+\ldots \\
& \left.\left.+\int_{{ }^{x_{1}}}^{x_{1}} \ldots \int_{{ }^{x_{n}}}^{x_{n}} \frac{\partial F(\xi)}{\partial \xi_{1}, \ldots, \xi_{n}} d \xi_{1} \ldots d \xi_{n}\right)-\mu\right)^{2} p(\mathbf{x}) d x_{1} \ldots d x_{n}
\end{aligned}
$$

$$
\begin{aligned}
\mu=\int_{-\infty}^{\infty} F(\mathbf{x}) & p(\mathbf{x}) d \mathbf{x}=F\left({ }^{\mathbf{c}} \mathbf{x}\right)+\sum_{i=1}^{n} \int_{-\infty}^{\infty} \int_{{ }^{\prime} x_{i}}^{x_{i}} \frac{\partial F(\xi)}{\partial \xi_{i}} d \xi_{i} p_{i}\left(x_{i}\right) d x_{i}+ \\
& +\sum_{1 \leq i<j \leq n} \int_{-\infty}^{\infty} \int_{-\infty}^{\infty} \int_{c_{x_{i}}}^{x_{i}} \int_{{ }^{\prime} x_{j}}^{x_{j}} \frac{\partial F(\xi)}{\partial \xi_{i}, \xi_{j}} d \xi_{i} d \xi_{j} p_{i j}\left(x_{i}, x_{j}\right) d x_{i} d x_{j}+\ldots \\
& +\int_{-\infty}^{\infty} \ldots \int_{-\infty}^{\infty} \int_{{ }^{x_{1}}}^{x_{1}} \ldots \int_{{ }^{x_{n}}}^{x_{n}} \frac{\partial f(\xi)}{\partial \xi_{1}, \ldots, \xi_{n}} d \xi_{1} \ldots d \xi_{n} p_{1 \ldots n}\left(x_{1}, \ldots, x_{n}\right) d x_{1} \ldots d x_{n}
\end{aligned}
$$

where $p_{i, \ldots, j}\left(x_{i}, \ldots, x_{j}\right)$ are the PDFs for the considered set of stochastic variables. It should be noted that the higher order moments cannot be computed as the superposition of the independent partial moments of the increment functions, i.e.

$$
\sigma^{2} \neq \sum_{k=1}^{2^{n}-1} \sigma_{k}^{2}
$$

The HDMR method and the associated sensitivity analysis theory and derivation are detailed in Kubicek et al. (2015). 


\subsubsection{HDMR Toy Problem}

For a practical insight into the theoretical basis presented thus far, a simple case problem is presented and discussed here. The properties of the method and the visualization of higher-order increment functions are demonstrated by using the following function:

$$
F\left(x_{1}, x_{2}, x_{3}\right)=0.5 x_{1}^{3}+0.5 x_{2}^{2}-e^{x_{1} x_{3}}
$$

where $x_{i}$ is a random variable with a uniform distribution and defined on the interval $[0,2]$. In this case, ${ }^{\mathbf{c}} \mathbf{x}=[1,1,1]^{T}$ and $F\left({ }^{\mathbf{c}} \mathbf{x}\right)=1-e=-1.7183$, and the general form of the Eq. 16 would be:

$$
\begin{array}{r}
F(\mathbf{x})-F\left({ }^{\mathbf{c}} \mathbf{x}\right)=\int_{{ }^{x_{1}}}^{x_{1}} \frac{\partial F(\xi)}{\partial \xi_{1}} d \xi_{1}+\int_{c_{x_{2}}}^{x_{2}} \frac{\partial F(\xi)}{\partial \xi_{2}} d \xi_{2}+\int_{c_{x_{3}}}^{x_{3}} \frac{\partial F(\xi)}{\partial \xi_{3}} d \xi_{3}+ \\
\int_{{ }^{x_{1}}}^{x_{1}} \int_{{ }^{x_{x_{2}}}}^{x_{2}} \frac{\partial F(\xi)}{\partial \xi_{1}, \xi_{2}} d \xi_{1} d \xi_{2}+\int_{{ }^{x_{1}}}^{x_{1}} \int_{{ }^{x_{x_{3}}}}^{x_{3}} \frac{\partial F(\xi)}{\partial \xi_{1}, \xi_{3}} d \xi_{1} d \xi_{3}+\int_{c_{x_{2}}}^{x_{2}} \int_{{ }^{x_{3}}}^{x_{3}} \frac{\partial F(\xi)}{\partial \xi_{2}, \xi_{3}} d \xi_{2} d \xi_{3}+ \\
\int_{{ }_{x_{x_{1}}}}^{x_{1}} \int_{{ }_{x_{x_{2}}}}^{x_{2}} \int_{{ }_{x_{3}}}^{x_{3}} \frac{\partial F(\xi)}{\partial \xi_{1}, \xi_{2}, \xi_{3}} d \xi_{1} d \xi_{2} d \xi_{3}
\end{array}
$$

or, more concisely

$$
F(\mathbf{x})-F\left({ }^{\mathbf{c}} \mathbf{x}\right)=d F_{x 1}+d F_{x 2}+d F_{x 3}+d F_{x 1, x 2}+d F_{x 1, x 3}+d F_{x 2, x 3}+d F_{x 1, x 2, x 3}
$$

The first order increment functions are established using samples on the given abscissas followed by addition of samples through adaptive sampling until convergence of the mean and standard deviation. The statistical properties for each increment function are established using a MC campaign on the corresponding surrogate, allowing to visualize the influence of the increment function on the final distribution. The evaluate and neglect approach correctly neglects all the higher order increment functions except the increment function $d F_{x 1, x 3}$. Eq. 29 then becomes:

$$
F(\mathbf{x})-F\left({ }^{\mathbf{c}} \mathbf{x}\right)=d F_{x 1}+d F_{x 2}+d F_{x 3}+d F_{x 1, x 3}
$$

The samples and surrogate models for the first order and non-neglected higher order increments functions are shown in Figure 3. The surrogate model functions fit through the samples for each increment function are 
picked from a database of model functions. The model function used for all increments functions in the toy problem is the multidimensional Lagrange interpolation. The partial histograms for the contributing increment functions are shown in Figures $4 \mathrm{a}-4 \mathrm{~d}$. The final overall distribution is computed as a sum of all the non negligible partial distributions and is shown in Figure 4 e. It can be seen that the body of the final distribution is dominated by contributions from the increment functions $\mathrm{dF}_{x 1}$ and $\mathrm{dF}_{x 3}$. An interesting contribution comes from the second order interaction $\mathrm{dF}_{x 1, x 3}$ that is mainly responsible for the heavy left tail on the final distribution. Note that the second order interaction $\left(\mathrm{dF}_{x 1, x 3}\right)$ is only active (is not 0 ) if both the first order increment functions $\left(\mathrm{dF}_{x 1}\right.$ and $\left.\mathrm{dF}_{x 3}\right)$ are active.

\subsubsection{Random Input Parameter Sampling}

Figure 5 summarizes the methodology with a flowchart representation. For this particular re-entry application we have two different $F(\mathbf{x})$ 's, namely the time-integrated longitude and latitude at ground impact. For each case, the $F(\mathbf{x})$ 's are decomposed into increment functions $\mathrm{d} F_{n}(\mathbf{x})$ as described in the previous section, where $n$ is the number of considered stochastic input parameters given in Table 1 along with the associated distribution types used for this work. For Gaussian distributions, the table provides the values for mean, standard deviation and the nominal values. For uniform distributions, the minimum, maximum, and nominal values are provided.

The surrogate models for each independent increment function are developed using an adaptive sampling strategy and sensitivity analysis to within predefined convergence residuals. The sensitivity analysis of the independent increment functions provide insight into the relevance of the higher order increment functions. Higher order increment functions with insensitive independent increment functions are deemed to have minor contribution and are neglected, contributing to the reduction of expensive BBM calls and, therefore, the computational cost. Additional test sampling of the stochastic input space is performed to determine the increment functions that can be further neglected, again saving expensive BBM calls towards surrogate model development. The developed surrogate models are sampled using MC to compute the statistical properties of each increment function. This allows to visualize through histograms the importance of each stochastic input variable and higher order interactions. The histograms of the independent increment functions will henceforth be also referred to as partial histograms.

Interpolation routines were developed for atmospheric temperature and 
density based on the temperature and density distributions given in Table 1 . The distribution data up to an altitude of $90 \mathrm{~km}$ is derived from the US Standard Atmosphere (1976). The mean value at $120 \mathrm{~km}$ was read off the US standard atmosphere table and the variation was assumed to be the same as at $90 \mathrm{~km}$. Figures 6 and 7 show 1000 samples drawn from the temperature and density interpolation routines, constrained to the data points, respectively. Each function call uses one such sample. Also shown in Figures 6 and 7 are the visual representations of temperature and density distributions, respectively, for the different altitudes [US Standard Atmosphere (1976)].

The atmosphere is assumed to be composed of $\mathrm{N}_{2}$ and $\mathrm{O}_{2}$ and the mole fraction of $\mathrm{N}_{2}$ is assumed to be uncertain. The heat capacity $\left(c_{p}\right)$ of the freestream air that has an effect on the computation of drag coefficient through the pressure behind the shock is also assumed to be uncertain. The flight path angle $(\gamma)$ of a re-entering object is considered uncertain with different distributions used for the 'shallow', 'normal', and 'steep' simulation cases. The re-entry speed $\left(V_{\infty}\right)$, mass of object $(m)$ and direction angle $(\chi)$ at 120 $\mathrm{km}$ are also considered uncertain. The central value of these parameters are based on the expected orbital and object characteristics with all uncertain variables considered to be statistically independent.

\section{Results and Discussion}

The longitudinal and lateral distributions of the impact location are computed using the following relation:

$$
y=F(\mathbf{x}) R e
$$

where $y$ represents two separate distributions in the longitudinal and lateral directions. These distributions correspond to two separate outputs of interest $F(\mathbf{x})$, namely the longitude and latitude angle difference between the entry point and the impact location, respectively. Re is the radius of the Earth. The relation in Eq. 31 is used to compute the contributions of each increment function to the longitudinal and lateral distributions, where the final distributions are computed as the sum of the contributions of the increment functions.

\subsection{Controlled 'Normal' Re-entry}

Table 2 and Table 3 give the sensitivity indices associated with the independent and higher order increment functions, respectively. Increment 
functions that have a sensitivity of less than $1 \%$ are neglected. The partial means represent the offset from the nominal value computed using the central value of the stochastic input distributions. The partial standard deviations are the contribution of the given increment function to the standard deviation of the overall distribution. The mean and variance sensitivity represent the normalized influence of the given stochastic input variable.

Table 2 highlights the parameters that strongly affect the distribution. The flight path angle $(\gamma)$ has the strongest effect on the longitudinal impact location distribution causing more than $75 \%$ of the total variation in the final distribution and a mean offset of more than $130 \mathrm{~km}$. The direction angle $(\chi)$ has the strongest effect on the lateral distribution with all other independent variables providing essentially no contribution. Table 3 gives the sensitivity indices associated with the higher order increment functions. In the longitudinal direction, an important contribution to the final distribution comes from the interaction $\mathrm{dF}_{\gamma, V_{\infty}}$. In the lateral direction, important contributions come from the interactions $\mathrm{dF}_{\gamma, \chi}, \mathrm{dF}_{V_{\infty}, \chi}$, and $\mathrm{dF}_{\gamma, V_{\infty}, \chi}$ with $\chi$ contributing to all interaction effects.

Figure 8 shows the partial histograms of the increment functions for the longitudinal impact distance. Figures $8 \mathrm{a}$ and $8 \mathrm{~b}$ show that the $\mathrm{dF}_{\gamma}$ and $\mathrm{dF}_{V_{\infty}}$ increment functions have parabolic shapes (i.e $\mathrm{x}^{2}$ ) resulting in an output that is always positive. This suggests that the same error in measuring higher velocities will lead to a larger uncertainty. Also, the size of the contribution suggests that these two variables are responsible for the overall shape of the final distribution. Figure $8 \mathrm{c}$ shows the partial histogram for the increment function $\mathrm{dF}_{\gamma, V_{\infty}}$.

Figure 9a shows the final distribution in the longitudinal direction derived as the sum of the distributions shown in Figure 8. The final distribution has a sharp peak that smoothly transitions into a long tail. The peak is a result of the second order functions for surrogate models of $\mathrm{dF}_{\gamma}$ and $\mathrm{dF}_{V_{\infty}}$ shown in Figures $8 \mathrm{a}$ and $8 \mathrm{~b}$, respectively. The long tail in the final distribution is a result of the surrogate model for the interaction function $\mathrm{dF}_{\gamma, V_{\infty}}$ shown in Figure 8c. The tail is caused by a steep ascent in the underling function in one of the corners of the given stochastic domain, i.e. to avoid such a strong tail, the input distributions need to be shortened from one side. The smooth decrease on the left side of the distribution $\mathrm{dF}_{\gamma, V_{\infty}}$ is responsible for smooth decrease on the left side in the final distribution (Figure 9a).

Results obtained with the HDMR approach are validated against MC analysis directly applied on the BBM using 1e 5 samples. Figures $9 \mathrm{~b}$ and $9 \mathrm{c}$ 
show the longitudinal distribution obtained using $\mathrm{MC}$ and the overlay of the HDMR and MC distributions, respectively. The histogram of the errors in the final PDF derived using HDMR in comparison with the MC solution for the longitudinal direction is given in Figure 9d. The small errors in Figure 9d and the statistics of the two distributions given in Table 4 suggest that the distributions are simply identical.

Figure 10 shows the partial histograms of the increment functions for the lateral impact distance. Figure 10a shows that the surrogate model for $\mathrm{dF}_{\chi}$ is a linear function with translation of the uniform input to output distribution. Important contributions are made by the interaction effects of $\mathrm{dF}_{\gamma, \chi}, \mathrm{dF}_{V_{\infty}, \chi}$, and $\mathrm{dF}_{\gamma, V_{\infty}, \chi}$. Both $\mathrm{dF}_{\gamma, \chi}$ and $\mathrm{dF}_{V_{\infty}, \chi}$ have a sharp peak around zero and smooth transitions to the tail on both sides. $\mathrm{dF}_{\gamma, V_{\infty}, \chi}$ exhibits a very sharp peak around zero with the distribution quickly converting to very thin but long tails on either side. The influence of the higher order interactions on the final distribution is well explained in the work of Kubicek et al. (2015).

For the final lateral distribution as shown in Figure 11a, the main contribution is from the direction angle as given in Table 2. The distribution exhibits a smooth Gaussian like transition from the tails to the peak where a plateau feature is quiet clearly visible. The smooth transition is contributed by $\mathrm{dF}_{\gamma, \chi}$ and $\mathrm{dF}_{V_{\infty}, \chi}$, whereas the plateau is derived from the uniform distribution of $\mathrm{dF}_{\chi}$. The very long tails are derived from $\mathrm{dF}_{\gamma, V_{\infty}, \chi}$. The MC lateral distribution is shown in Figure 11b. The statistics in Table 4 combined with the overlay shown in Figure 11c, and the final PDF error histogram shown in Figure 11d validate the HDMR methodology. Figure 12 shows that comparison of the 2-Dimensional distribution used in the calculation of the total impact area. The comparison again confirms identical distributions with the interaction effects providing the 2-D spread.

In addition to the case with 16 random variables, three additional cases were simulated: 1 ) a case with 12 random variables accounting for uncertainty in atmospheric conditions only, 2) a case with 13 random variables accounting for uncertainty in atmospheric conditions $+\gamma$, and 3) a case with 15 random variables accounting for uncertainty in atmospheric conditions $+\gamma+V_{\infty}$ $+m$. These cases provide an insight into the effect of missing important stochastic variables. It is obvious and expected that not considering the direction angle as uncertain and not modeling for fragmentation will collapse the lateral distribution down to the deterministic solution in that direction. Therefore, we examine the other cases on the basis of the final distribution in the longitudinal direction. 
Tables 2 and 3 are still representative of the sensitivities for all the cases with small differences arising as a result of additional uncertain variables into the problem. The convergence of the method is connected to the final statistical characteristics, which changes with the additional uncertain variables. Therefore, the relative sensitivity remains the same while the independent sensitivities are scaled based on the number of uncertain variables. Table 5 gives the number of expensive BBM calls required to develop the surrogate models for the different cases. As expected, the number of function calls increases with addition of uncertain input parameters, however, in all the different cases the HDMR methodology reduces the number of expensive BBM calls by close to 3 orders of magnitude. It should be noted that the learning process, requiring just a few seconds, has a computational cost that is negligible with respect to the cost of obtaining samples from the BBM. This allows the simulations to be run on a regular desktop machine and does not require expensive time on supercomputers.

Figure 13 shows the distributions for the impact distance from the entry point for the three additional cases. Figure 13a shows that accounting for only the atmospheric uncertainties results in (close to) the coveted Gaussian distribution spread over a little more than $100 \mathrm{~km}$. Figure 13b shows that when also taking into account uncertainty in $\gamma$, the distribution departs significantly from the Gaussian with the spread growing to near $2400 \mathrm{~km}$. Figure $13 \mathrm{c}$ shows that accounting for the uncertainty in $V_{\infty}$ can further influence the ground impact distribution with the spread now rising to over 4500 $\mathrm{km}$. The second order velocity effects through drag and velocity interaction effects, as derived from the sensitivity data in Tables 2 and 3, results in a slender but elongated tail. Unlike a Gaussian distribution, the mean values of the distributions for the $13 \mathrm{D}$ an $15 \mathrm{D}$ cases are significantly offset from the peak of the distributions. For reference, the deterministic impact location in the longitudinal direction corresponding to the central values of the distributions for stochastic input variables lies at approximately $2400 \mathrm{~km}$. As can be seen, the peaks of the distributions for all the cases are significantly offset from the deterministic solution.

\subsection{Uncontrolled 'Shallow' and Controlled 'Steep' Re-entry}

Figures 14a and 14b show the comparison of distributions derived from HDMR and MC for the un-controlled 'shallow' re-entry case in the longitudinal and lateral directions, respectively. Figures $14 \mathrm{c}$ and $14 \mathrm{~d}$ show the PDF error histograms for the case of uncontrolled shallow re-entry case in the 
longitudinal and lateral directions, respectively. The distribution in the longitudinal direction again is driven mainly by $\gamma$ and $V_{\infty}$. The HDMR method captures well the shape of the distribution except for the small, sharp peak towards the tail end of the distribution. The peak is a result of a small oscillation in the MC distribution that is not captured by the increment function of $V_{\infty}$ as seen in Figure 14e. Unfortunately, such problems (capturing small deviations from the interpolation function) cannot be solved in general and are inherent to numerical integration and interpolation methods and form as such a limitation of the HDMR method. Nevertheless, there is very good agreement between the distributions both in the longitudinal and lateral directions (as evident from the small errors in Figures 14c and 14d and the statistics in Table 4) with the HDMR requiring only 127 samples, i.e. expensive BBM calls.

Figures $15 \mathrm{a}$ and $15 \mathrm{~b}$ show the comparison of distributions derived from HDMR and MC for the controlled 'steep' re-entry case in the longitudinal and lateral directions, respectively. Figures $15 \mathrm{c}$ and $15 \mathrm{~d}$ show the PDF error histograms for the case of controlled steep re-entry case in the longitudinal and lateral directions, respectively. Just like in the case of the un-controlled 'shallow' case, the HDMR distributions in both the longitudinal and lateral direction match very well with those derived with MC, with the HDMR requiring only 145 expensive BBM calls.

Several interesting observations can be made on comparing the longitudinal and lateral distributions for the different cases (un-controlled 'shallow' and controlled 'normal' and 'steep' cases): 1) the longitudinal distribution seems to approach a Gaussian with steeper $\gamma$, which is expected because as the re-entry occurs at steeper angles, the amount of time the object spends traversing the atmosphere is drastically reduced and so is the chance for the other uncertainties (especially atmospheric) to have an effect. 2) The longitudinal impact distribution for the 'shallow' case is not as wide as that for the 'normal' case because of a smaller input distribution of $\gamma$. Both distributions have the same larger boundary because the input $\gamma$ distributions for both cases have a common boundary at zero degrees that corresponds to the largest impact distance from the entry point. The longitudinal distribution for the controlled 'steep' case has a very small spread with large impact probabilities close to the mean value of the distribution and has no overlap with the other two cases because the object falls well short of the impact locations for the other cases due to the high $\gamma$ values. 3) The lateral impact distributions for the 'shallow' and 'normal' cases have the same spread 
(boundary values) corresponding to the common boundary value of zero degrees in the input distributions for $\gamma$ while the distribution is much narrower for the 'steep' case. Increasing $\gamma$ results in large impact probabilities close to the mean value of the distribution.

\section{Conclusion}

The objective of this study was to gain an understanding for the effects of uncertainties on the re-entry trajectory and impact location and to introduce a novel and efficient methodology for probabilistic modeling of the atmospheric re-entry. Current re-entry modeling tools perform the analysis in a deterministic sense and do not include any uncertainty treatment. This work presents progress towards incorporating uncertainty treatment into the modeling of atmospheric re-entry of space debris using a recently developed novel high-dimensional derivative based uncertainty quantification approach. Validation of the results obtained from the High Dimensional Model Representation methodology with a Monte Carlo analysis is also presented.

Re-entry simulations with initial conditions corresponding to a circular orbit are performed for a spherical object accounting for both aleatoric and epistemic uncertainties. Uncertainties affecting atmospheric properties - such as temperature, density, composition, and heat capacity - initial conditions - such as speed, flight path angle, and direction angle - as well as object mass are considered. Three different re-entry cases are simulated to test and validate the applicability of the developed method to both controlled and un-controlled re-entry scenarios: 1) un-controlled 'shallow' re-entry, 2) controlled 'normal' re-entry, and 3) controlled 'steep' re-entry. The results show that the High Dimensional Model Representation based approach is applicable to all re-entry scenarios.

Multiple sub-cases are also run for the controlled 'normal' re-entry to develop an understanding of the effects of the uncertain parameters on the final ground impact distribution. A total of four cases are simulated for the controlled 'normal' re-entry: 1) a case with 12 random variables accounting for uncertainty in atmospheric conditions only, 2) a case with 13 random variables accounting for uncertainty in atmospheric conditions + flight path angle 3) a case with 15 random variables accounting for uncertainty in atmospheric conditions + flight path angle + re-entry speed + object mass, and 4$)$ a case with all 16 random variables that also includes, in addition to those in the $15 \mathrm{D}$ case, the direction angle. 
Accounting for only the atmospheric uncertainties results in (close to) the coveted Gaussian distribution for the impact distance in the longitudinal direction spread over a little more than $100 \mathrm{~km}$. However, accounting for uncertainties in the object properties results in distributions that are significantly different from a Gaussian. Taking into account the flight path angle (case 2), the distribution spread increases to near $2400 \mathrm{~km}$ which further increases to over $4500 \mathrm{~km}$ accounting for uncertainties in the re-entry speed at $120 \mathrm{~km}$ (case 3 and 4). For reference, the deterministic impact location in the longitudinal direction corresponding to the central values of the distributions for the stochastic input variables lies at $2376 \mathrm{~km}$. As observed, the peaks of the distributions for all the cases are significantly offset from the deterministic solution. The lateral distribution when taking into account the direction angle (case 4) has a Gaussian-like smooth transition from the tail towards the peak where a plateau feature is observed and is spread over close to $40 \mathrm{~km}$.

The novelty of the high-dimensional technique implemented is that through the formulation of the problem, it allows to visualize through partial histograms and sensitivity indices the effect and importance of each independent random variable and their combinations on the output parameter of interest. Results show that the uncertainty in the longitudinal direction is dominated by the flight path angle causing more than $75 \%$ of the variance followed by the re-entry speed as well as the interaction effects between the two. The two parameters independently and through interactions make up for more than $99.5 \%$ of the variance. Uncertainty in the lateral distribution is dominated by the direction angle with close to $90 \%$ variance contribution and the rest caused by the interactions between the direction angle, flight path angle, and re-entry speed.

The next step in this research stream will be the integration of the developed and tested uncertainty treatment approach into the Free Open Source Tool for Re-entry of Asteroids and Space Debris, FOSTRAD, and its application to demise analysis and design for demise processes.

\section{Acknowledgement}

Funding for Piyush Mehta is provided by the European Commission through the Marie Curie Initial Training Network (ITN) STARDUST under grant number 317185. Partial support for Martin Kubicek is provided by 'OPTIMAD Engineering Srl'. The authors would like to acknowledge the use of the 
EPSRC funded ARCHIE-WeSt High Performance Computer (www.archiewest.ac.uk), EPSRC grant no. EP/K000586/1.

\section{References}

NASA-STD-8719.14. Process for Limiting Orbital Debris, May 2012, Revision A Change 1.

Requirements on Space Debris Mitigation for ESA Projects, European Space Agency, April 2008.

Rochelle, W. C., Kirk, B. S., and Ting B. C., User's Guide for Object Reentry Analysis Tool (ORSAT), JSC-28742, Version 5.0, Vol. 1., NASA Lyndon B. Johnson Space Center, 1999.

Koppenwallner, G., Fritsche, B., Lips, T., and Klinkrad, H., SCARAB A multi-disciplinary code for destruction analysis of spacecraft during reentry, Proceeding of the 5th European Symposium on Aerothermodynamics of Space Vehicles, Cologne, Germany, Nov 2005.

Martin, C., Brandmueller, C., Bunte, K., et al., A Debris Risk Assessment Tool Supporting Mitigation Guidelines, Proceeding of the 4th European Conference on Space Debris, ESA SP-587, ESA/ESOC, Darmstadt, Germany, April 2005.

Parigini C., Fuentes I. P., Ramos R. H., et al., Debris tool and its use in mission analysis activities, Proceeding of the 8th ESA symposium on aerothermodynamics of space vehicles, Lisbon, Portugal, March 2015.

Lips T. and Fritsche B., A comparison of commonly used re-entry analysis tools, Acta Astronautica, Vol. 57, 2005, pp. $312-323$.

Saltelli, A., Tarantola, S., Campolongo, F., et al., Sensitivity Analysis in Practice: A Guide to Assessing Scientific Models, John Wiley and sons, 2004.

Saltelli, A., Ratto, M., Andres, et al. Global Sensitivity Analysis: The Primer, John Wiley and sons, January 2008. 
Kleijnen, J. P. C., and Helton, J. C., Statistical analyses of scatter-plots to identify important factors in large-scale simulations, 1: Review and comparison of techniques, Reliability Engineering and System Safety, Vol. 65, No. 2, 1999, pp. 147-185.

Kleijnen, J. P. C., and Helton, J. C., Statistical analyses of scatter-plots to identify important factors in large-scale simulations, 2: robustness of techniques, Reliability Engineering and System Safety, Vol. 65, No. 2, 1999, pp. 187197.

Eldred, M. and Burkardt, J., Comparison of non-intrusive polynomial chaos and stochastic collocation methods for uncertainty quantification, 47th AIAA Aerospace Sciences Meeting including The New Horizons Forum and Aerospace Exposition, Orlando, Florida, January 2009.

Eldred, M., Recent advances in non-intrusive polynomial chaos and stochastic collocation methods for uncertainty analysis and design, 50th AIAA /ASME / ASCE / AHS / ASC Structures, Structural Dynamics, and Materials Conference, Palm Springs, California, May 2009.

Wiener, N., The homogeneous chaos, America Journal of Mathematics, Vol. 60, NO. 4, October 1938, pp. 897-936.

Hosder, S., Walters, R., and Perez, R., A non-intrusive polynomial chaos method for uncertainty propagation in cfd simulations, 44th AIAA Aerospace Sciences Meeting and Exhibit, Reno, Nevada, January 2006.

Eldred, M. S., Webster, C. G., and Constantine, P. G., Evaluation of nonintrusive approaches for wiener-askey generalized polynomial chaos, 49th AIAA /ASME / ASCE / AHS / ASC Structures, Structural Dynamics, and Materials Conference, 2008.

Cheng, H. and Sandu, A., Collocation least-squares polynomial chaos method, Proceedings of the 2010 Spring Simulation Multi-conference, San Diego, CA, 2010.

Togawa, K., Benigni, A., and Monti, A., Advantages and challenges of non intrusive polynomial chaos theory, In: Crosbie, R. (Ed.), Proceedings of the 2011 Grand Challenges on Modeling and Simulation Conference, Vista, CA, June 2011, pp. 30-35. 
Branicki, M. and Majda, A., Fundamental limitations of polynomial chaos for uncertainty quantification in systems with intermittent instabilities, Communications in Mathematical Sciences, Vol. 11, 2013.

Fang, K. T., Li, R., and Sudjianto, A., Design and Modeling for Computer Experiments, Chapman and Hall/CRC press, New York, 2006.

Lee, J. and Kwon, J. H., On the use of kriging in the interpolation of fluidstructure interaction analysis, Japan society of computational fluid dynamics, Vol. 16, 2008.

Forrester, A. I. J., Sobester, A., and Keane, A. J., Engineering Design via Surrogate Modelling, A John Wiley and Sons, Ltd., 2008.

Sudret, B., Global sensitivity analysis using polynomial chaos expansions, Reliability Engineering and system safety, 2008.

Bellman, R. E., Adaptive Control Processes: A Guided Tour, Princeton University Press, New Jersey, NJ, 1961.

Mehta, P. M., Walker, A., Lawrence, E., et al., Modeling satellite drag coefficients with response surfaces, Advances in Space Research, Vol. 54, No. 8, 15 October 2014, pp. 1590-1607.

Chen, R. B., Hsieh, D. N., Hung, Y., and Wang, W., Optimizing latinhypercube designs by particle swarm, Statistics and Computing, Vol. 23, September 2013, pp. 663-676.

Hosder, S., Walters, R. W., and Balch, M., Efficient sampling for nonintrusive polynomial chaos applications with multiple uncertain input variables, 48th AIAA / ASME / ASCE / AHS / ASC Structures, Structural Dynamics, and Materials Conference, Honolulu, HI, April 2007.

Gerstner, T. and Griebel, M., Numerical integration using sparse grids, Numerical Algorithms, Vol. 18, 1998, pp. 209-232.

Barthelmann, V., Novak, E., and Ritter, K., High dimensional polynomial interpolation on sparse grids, Advances in Computational Mathematics, Vol. 12, 2000, pp. 273-288.

Pfluger, D., Spatially Adaptive Sparse Grids for High-Dimensional problem, Verlag Dr. Hut, Munchen, 2010. 
Kubicek, M., Minisci, E., and Cisternino, M., High dimensional sensitivity analysis using surrogate modeling and High Dimensional Model Representation, International Journal for Uncertainty Quantification, Vol. 5(5), 2015 .

Mehta, P. M., Minisci, E., and Vasile, M., Break-up Modeling and Trajectory Simulation under Uncertainty for Asteroids, Proceeding of the 4th IAA Planetary Defense Conference, Frascati, Roma, 13-17, April 2015.

Mehta, P. M., Kubicek, M., Minisci, E., et al., Surrogate Model for Probabilistic modeling of Atmospheric Entry for small NEO's, Proceedings of the 26th AAS/AIAA Spaceflight Mechanics, Napa, CA, AAS 16-245, 14-18 February 2016.

Mehta, P. M., Minisci, E., Vasile, M., et al., Sensitivity Analysis towards Probabilistic Re-Entry Modeling of Spacecraft and Space Debris, Proceeding of the AIAA Modeling and Simulation Technologies Conference, AIAA 2015-3098, Dallas, TX, June 2015.

Mehta, P. M., Arnao G. B., Bonetti, D., et al., Computer Graphics for Space Debris, Proceedings of the 6th International Conference on Astrodynamics Tools and Techniques, Darmstadt, Germany, 14-17 March 2016.

Newton, I., Principia - Mote's Translation Revised, University of California Press, 1946.

Schaaf, S. A., and Chambre, P. L., High Speed Aerodynamics and Jet Propulsion, ch. Flow of Rarefied Gases, Princeton Univ. Press, Princeton, NJ: 1958, pp. $1-55$.

Mehta, P. M., Minisci, E., Vasile, M., et al., An Open Source Hypersonic Aerodynamic and Aerothermo-dynamic Modeling Tool, Proceeding of the 8th European Symposium on Aerothermodynamics of Space Vehicles, Lisbon, Portugal, March 2015.

U.S. Standard Atmosphere, 1976, Tech. Rep. NOAA-S/T 76-1562, National Oceanic and Atmospheric Administration, Washington, DC: U.S. Government Printing Office, 1976. 
Gaoa, Z., and Hesthavenb, J. S., On ANOVA expansions and strategies for choosing the anchor point, Applied Mathematics and Computation, Vol. 217(7), 2010. 


\section{Appendix: Tables and Figures}

Table 1: Distribution properties of the stochastic input variables

\begin{tabular}{|c|c|c|c|c|c|}
\hline Variable & Description [Units] & Type & $\mu$ & $\sigma$ & Central Value \\
\hline$T_{0}$ & Temperature at $0 \mathrm{~km}[\mathrm{~K}]$ & Gumbell & 280.0 & 16.667 & 280.0 \\
\hline$T_{20}$ & Temperature at $20 \mathrm{~km}[\mathrm{~K}]$ & Gumbell & 218.0 & 7.333 & 218.0 \\
\hline$T_{50}$ & Temperature at $50 \mathrm{~km}[\mathrm{~K}]$ & Landau & 252.0 & 16.667 & 252.0 \\
\hline$T_{70}$ & Temperature at $70 \mathrm{~km}[\mathrm{~K}]$ & Landau & 187.0 & 24.0 & 187.0 \\
\hline$T_{90}$ & Temperature at $90 \mathrm{~km}[\mathrm{~K}]$ & Gaussian & 185.0 & 25.0 & 185.0 \\
\hline$T_{120}$ & Temperature at $120 \mathrm{~km}[\mathrm{~K}]$ & Gaussian & 360.0 & & 360.0 \\
\hline$\rho_{0}$ & Density at $0 \mathrm{~km}\left[\mathrm{~kg}-\mathrm{m}^{-3}\right]$ & Gaussian & 1.225 & $8.167 \mathrm{e}-2$ & 1.225 \\
\hline$\rho_{40}$ & Density at $40 \mathrm{~km}\left[\mathrm{~kg}-\mathrm{m}^{-3}\right]$ & Gaussian & $4.0 \mathrm{e}-3$ & $5.330 \mathrm{e}-4$ & $4.0 \mathrm{e}-3$ \\
\hline$\rho_{90}$ & Density at $90 \mathrm{~km}\left[\mathrm{~kg}-\mathrm{m}^{-3}\right]$ & Gaussian & $3.416 \mathrm{e}-6$ & $5.693 \mathrm{e}-7$ & $3.416 \mathrm{e}-6$ \\
\hline \multirow[t]{2}{*}{$\rho_{120}$} & Density at $120 \mathrm{~km}\left[\mathrm{~kg}-\mathrm{m}^{-3}\right]$ & Gaussian & $2.222 \mathrm{e}-8$ & $3.703 \mathrm{e}-9$ & $2.222 \mathrm{e}-8$ \\
\hline & & & Min & $\operatorname{Max}$ & \\
\hline$\overline{X_{N_{2}}}$ & Percentage of $N_{2}[\%]$ & Uniform & 0.784 & 0.816 & 0.8 \\
\hline$c_{p}$ & Heat Capacity $\left[\mathrm{J}-\mathrm{K}^{-1}\right]$ & Uniform & 1304.35 & 1441.65 & 1373 \\
\hline \multirow[t]{4}{*}{$\gamma$} & Flight Path Angle [deg] & & & & \\
\hline & Un-controlled 'Shallow' & Uniform & 0.0 & -0.1 & -0.05 \\
\hline & Controlled 'Normal' & Uniform & 0.0 & -2.5 & -1.25 \\
\hline & Controlled 'Steep' & Uniform & -4.5 & -5.5 & -5.0 \\
\hline$V_{\infty}$ & Re-entry Speed $\left[\mathrm{m}-\mathrm{s}^{-1}\right]$ & Uniform & 7410.0 & 7790.0 & 7600.0 \\
\hline$m$ & Mass of Debris [kg] & Uniform & 243.75 & 256.25 & 250 \\
\hline$\chi$ & Direction Angle [deg] & Uniform & 87.5 & 92.5 & 90.0 \\
\hline
\end{tabular}


Table 2: Sensitivity Characteristics of the Independent Increment Functions for the 16D case

\begin{tabular}{|c|c|c|c|c|}
\hline Variable & Partial $\mu[\mathrm{km}]$ & Partial $\sigma[\mathrm{km}]$ & $\mu$ Sensitivity & $\sigma^{2}$ Sensitivity \\
\hline \multicolumn{5}{|c|}{ Longitudinal } \\
\hline$T_{0}$ & $1.611 \mathrm{e}-05$ & $6.066 \mathrm{E}-05$ & $8.127 \mathrm{e}-08$ & $7.663 \mathrm{e}-15$ \\
\hline$T_{20}$ & $-6.744 \mathrm{e}-08$ & $1.165 \mathrm{E}-03$ & $3.404 \mathrm{e}-10$ & $2.828 \mathrm{e}-12$ \\
\hline$T_{50}$ & $-5.634 \mathrm{e}-05$ & $1.909 \mathrm{E}-02$ & $2.843 \mathrm{e}-07$ & $7.588 \mathrm{e}-10$ \\
\hline$T_{70}$ & $-2.684 \mathrm{e}-04$ & $5.627 \mathrm{E}-03$ & $1.354 \mathrm{e}-06$ & $6.596 \mathrm{e}-11$ \\
\hline$T_{90}$ & $-6.870 \mathrm{e}-05$ & $1.827 \mathrm{E}-03$ & $3.467 \mathrm{e}-07$ & $6.956 \mathrm{e}-12$ \\
\hline$T_{120}$ & $1.887 \mathrm{e}-06$ & $7.256 \mathrm{E}-04$ & $9.522 \mathrm{e}-09$ & $1.097 \mathrm{e}-12$ \\
\hline$\rho_{0}$ & $1.006 \mathrm{e}-08$ & $2.449 \mathrm{E}-07$ & $5.077 \mathrm{e}-11$ & $1.249 \mathrm{e}-19$ \\
\hline$\rho_{40}$ & $9.910 \mathrm{e}-01$ & $1.214 \mathrm{E}+01$ & $5.001 \mathrm{e}-03$ & $3.067 \mathrm{e}-04$ \\
\hline$\rho_{90}$ & $1.029 \mathrm{e}+00$ & $1.338 \mathrm{E}+01$ & $5.194 \mathrm{e}-03$ & $3.731 \mathrm{e}-04$ \\
\hline$\rho_{120}$ & $5.036 \mathrm{e}-02$ & $1.129 \mathrm{E}+00$ & $2.541 \mathrm{e}-04$ & $2.653 \mathrm{e}-06$ \\
\hline$X_{N_{2}}$ & $-1.281 \mathrm{e}-06$ & $8.730 \mathrm{E}-04$ & $6.465 \mathrm{e}-09$ & $1.588 \mathrm{e}-12$ \\
\hline$c_{p}$ & $-4.215 \mathrm{e}-03$ & $2.208 \mathrm{E}-01$ & $2.127 \mathrm{e}-05$ & $1.015 \mathrm{e}-07$ \\
\hline$\gamma$ & $1.316 \mathrm{e}+02$ & $6.057 \mathrm{E}+02$ & $6.644 \mathrm{e}-01$ & $7.642 \mathrm{e}-01$ \\
\hline$V_{\infty}$ & $2.811 \mathrm{e}+01$ & $2.002 \mathrm{E}+02$ & $1.419 \mathrm{e}-01$ & $8.348 \mathrm{e}-02$ \\
\hline$m$ & $-2.443 \mathrm{e}-02$ & $2.876 \mathrm{E}+00$ & $1.233 \mathrm{e}-04$ & $1.723 \mathrm{e}-05$ \\
\hline$\chi$ & $-6.745 \mathrm{e}-01$ & $6.047 \mathrm{E}-01$ & $3.404 \mathrm{e}-03$ & $7.616 \mathrm{e}-07$ \\
\hline \multicolumn{5}{|c|}{ Lateral } \\
\hline$\chi$ & $-6.083 e-03$ & $5.464 \mathrm{e}+01$ & $9.292 \mathrm{e}-02$ & $8.968 \mathrm{e}-01$ \\
\hline
\end{tabular}

Table 3: Sensitivity Characteristics of the higher order increment Functions for the 16D case.

\begin{tabular}{|c|c|c|c|c|}
\hline Variable & Partial $\mu[\mathrm{km}]$ & Partial $\sigma[\mathrm{km}]$ & $\mu$ Sensitivity & $\sigma^{2}$ Sensitivity \\
\hline \multicolumn{5}{|c|}{ Longitudinal } \\
\hline$\gamma, V_{\infty}$ & $3.562 \mathrm{e}+01$ & $2.698 \mathrm{e}+02$ & $1.798 \mathrm{e}-01$ & $1.516 \mathrm{e}-01$ \\
\hline \multicolumn{5}{|c|}{ Lateral } \\
\hline$\gamma, \chi$ & $4.680 \mathrm{e}-02$ & $1.776 \mathrm{e}+01$ & $6.564 \mathrm{e}-01$ & $9.248 \mathrm{e}-02$ \\
\hline$V_{\infty}, \chi$ & $1.127 \mathrm{e}-02$ & $5.516 \mathrm{e}+00$ & $1.581 \mathrm{e}-01$ & $8.924 \mathrm{e}-03$ \\
\hline$\gamma, V_{\infty}, \chi$ & $5.460 \mathrm{e}-03$ & $8.842 \mathrm{e}+00$ & $7.657 \mathrm{e}-02$ & $2.293 \mathrm{e}-02$ \\
\hline
\end{tabular}


Table 4: Validation statistics for the HDMR compared with Monte Carlo.

\begin{tabular}{lcccc}
\hline \hline \multicolumn{2}{c}{ Longitudinal } & Lateral \\
\hline \hline Mean [km] & Std. Deviation [km] & Mean [km] & Std. Deviation [km] \\
HDMR & $2.330 \mathrm{e}+03$ & $7.785 \mathrm{e}+02$ & $5.664 \mathrm{e}-02$ & $6.495 \mathrm{e}+01$ \\
Monte Carlo & $2.328 \mathrm{e}+03$ & $7.733 \mathrm{e}+02$ & $5.085 \mathrm{e}-02$ & $6.418 \mathrm{e}+01$ \\
\hline
\end{tabular}

Table 5: Number of expensive BBM calls for model development with HDMR.

\begin{tabular}{lcccc}
\hline \hline Case & $12 \mathrm{D}$ & $13 \mathrm{D}$ & $15 \mathrm{D}$ & $16 \mathrm{D}$ \\
\# of function Calls & 57 & 65 & 84 & 174 \\
\hline \hline
\end{tabular}




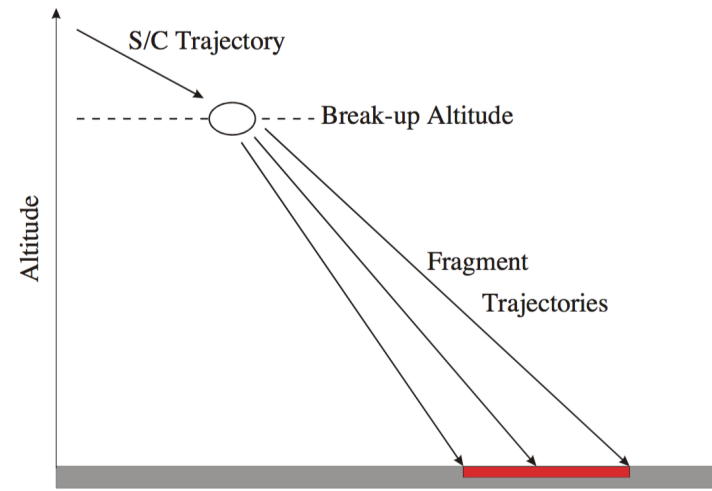

Impact Footprint

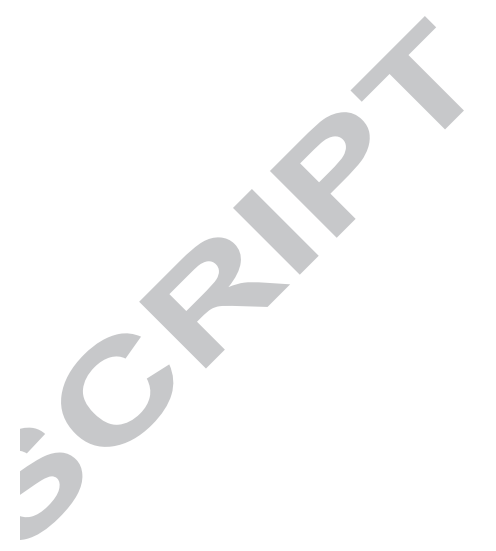

Figure 1: Concept behind an object-oriented tool [Lips and Fritsche (2005)].

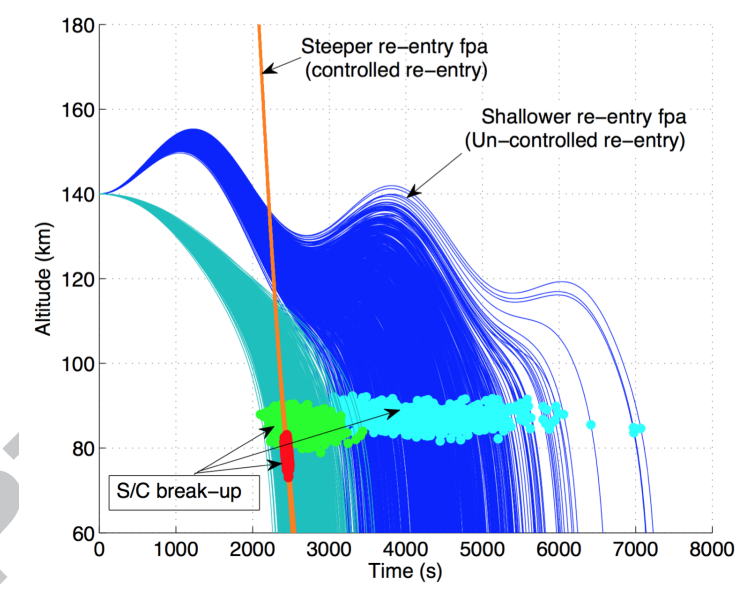

Figure 2: Monte Carlo analysis and the concept behind an object-oriented tool [Parigini et al. (2015)]. fpa $=$ flight-path angle. 


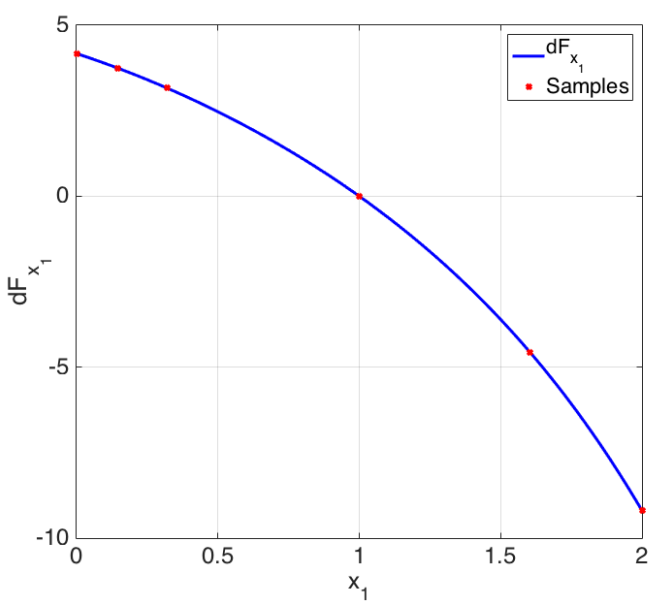

(a)

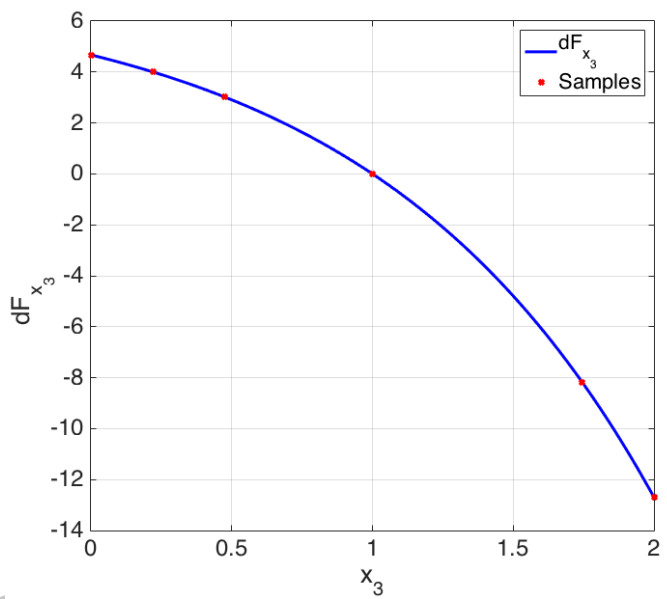

(c)

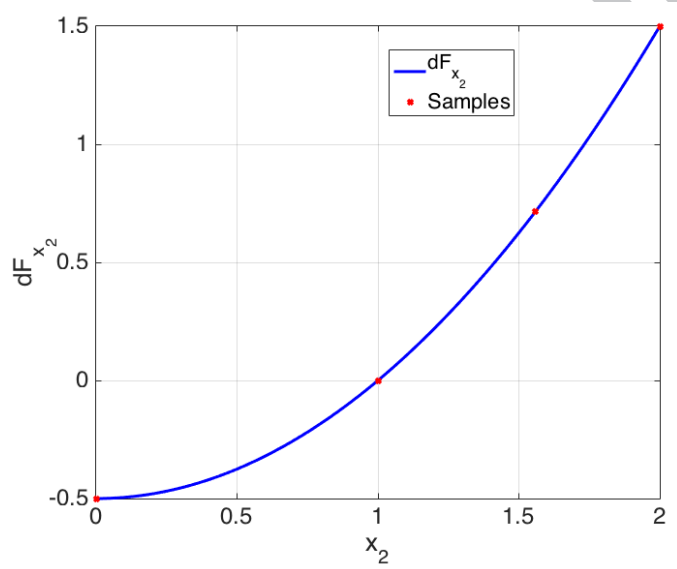

(b)

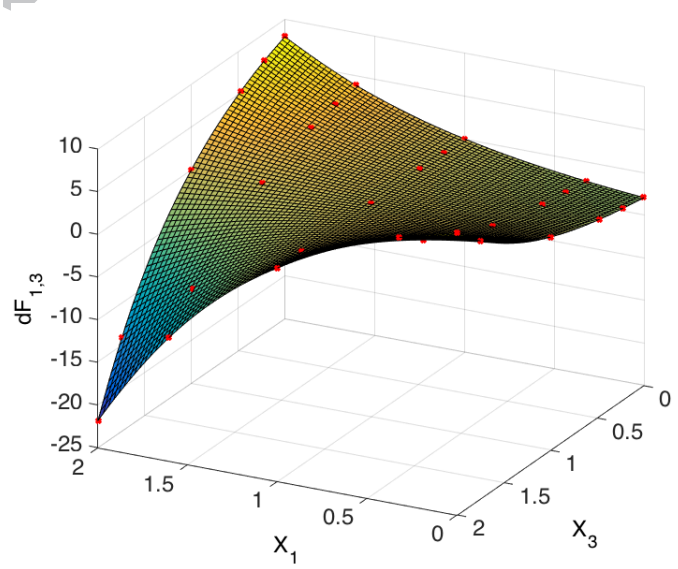

(d)

Figure 3: Surrogates for first and higher order increments functions developed through adaptive sampling 


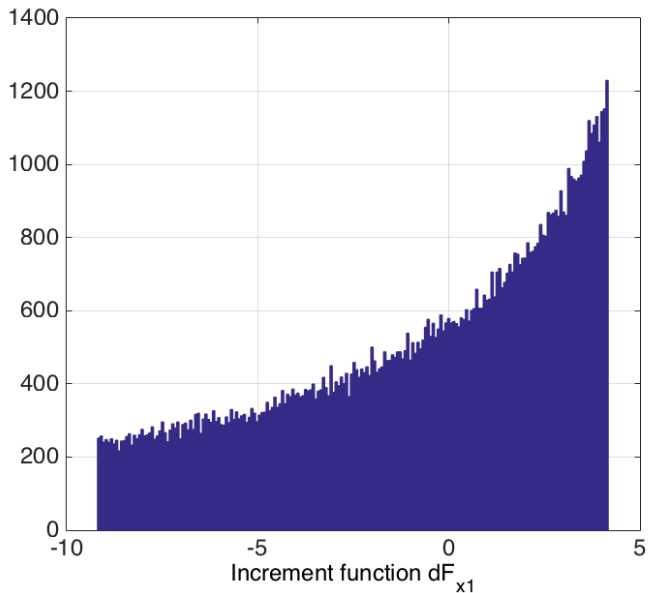

(a)

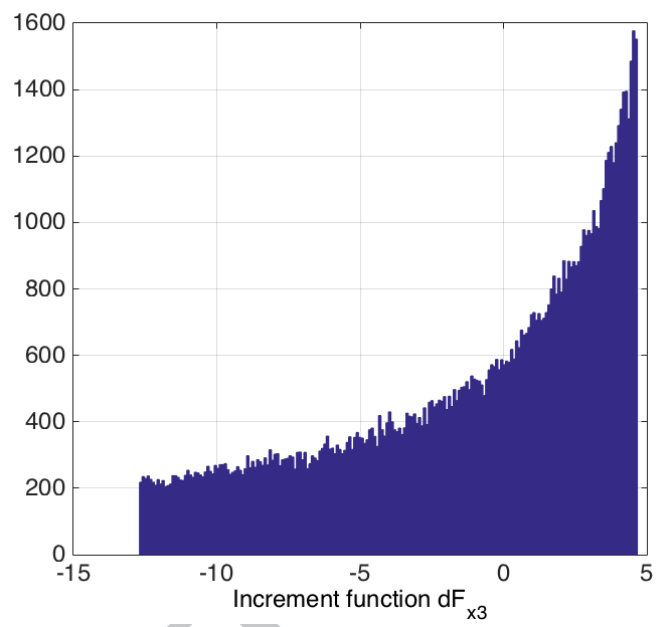

(c)

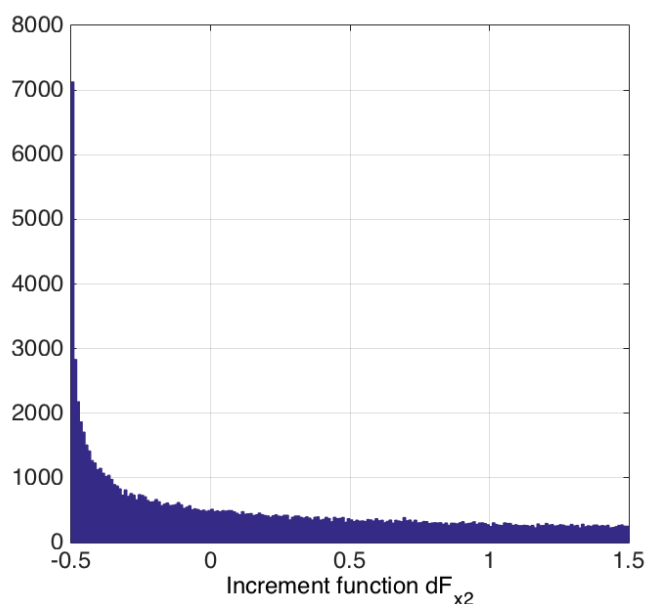

(b)

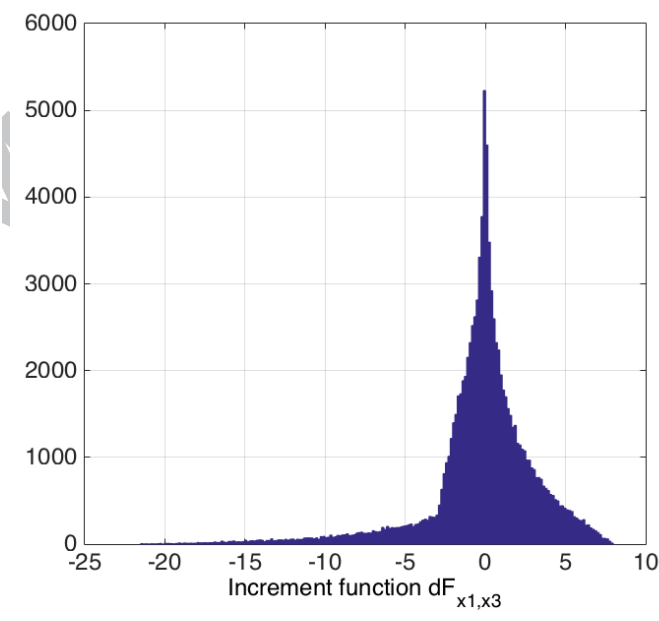

(d)

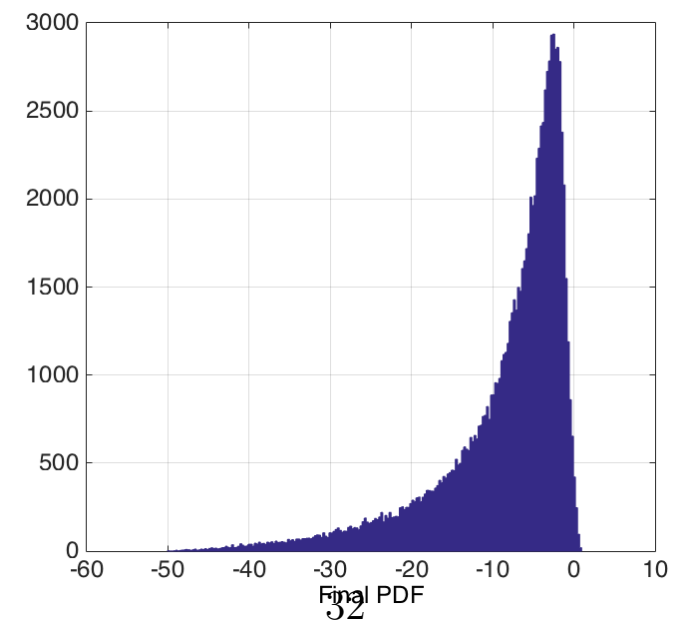

(e)

Figure 4: Partial histograms of the increment functions for the toy problem. 


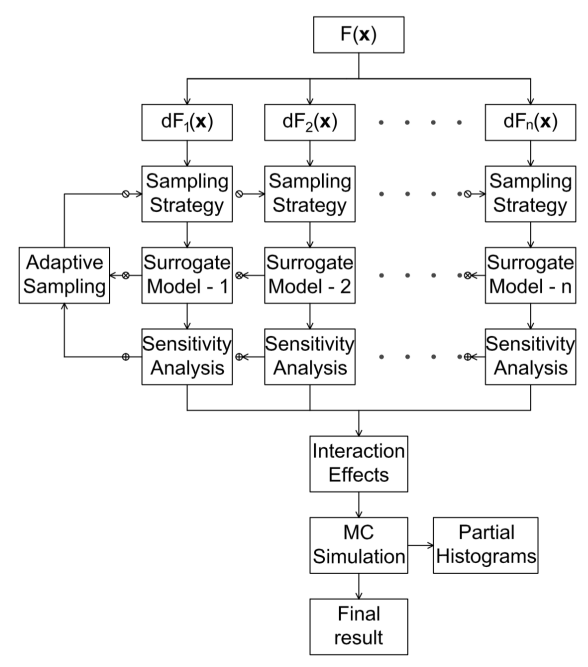

(a)

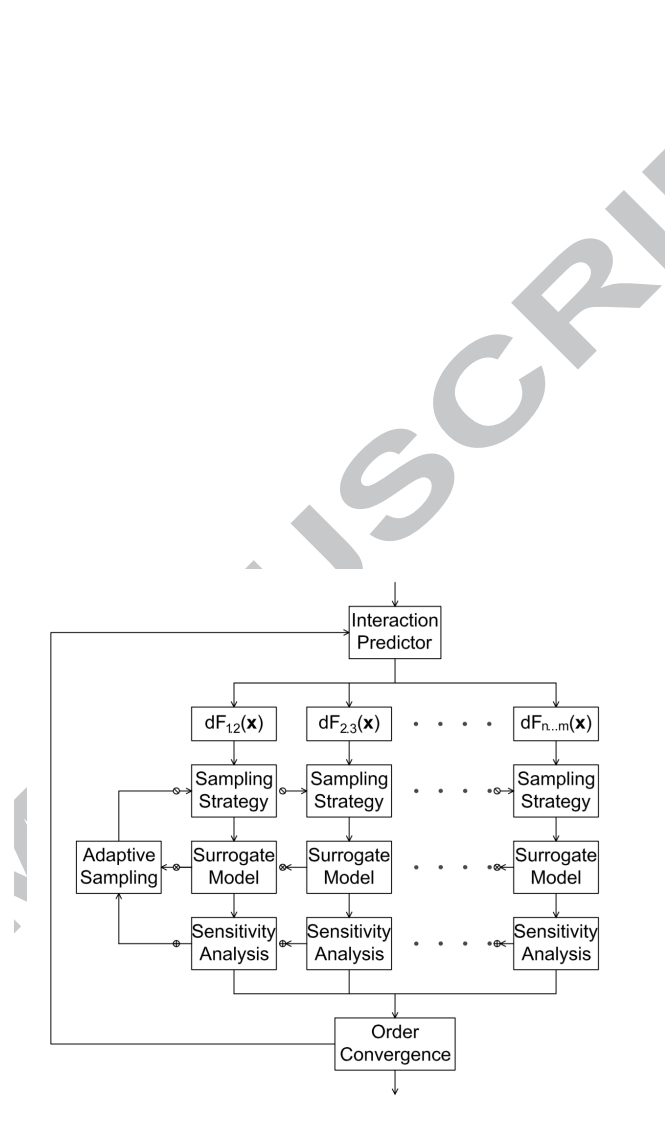

(b)

Figure 5: (a) Characteristic flowchart for the Uncertainty Treatment (b) Expansion of the "Interaction Effects" block in the flowchart 5a. 

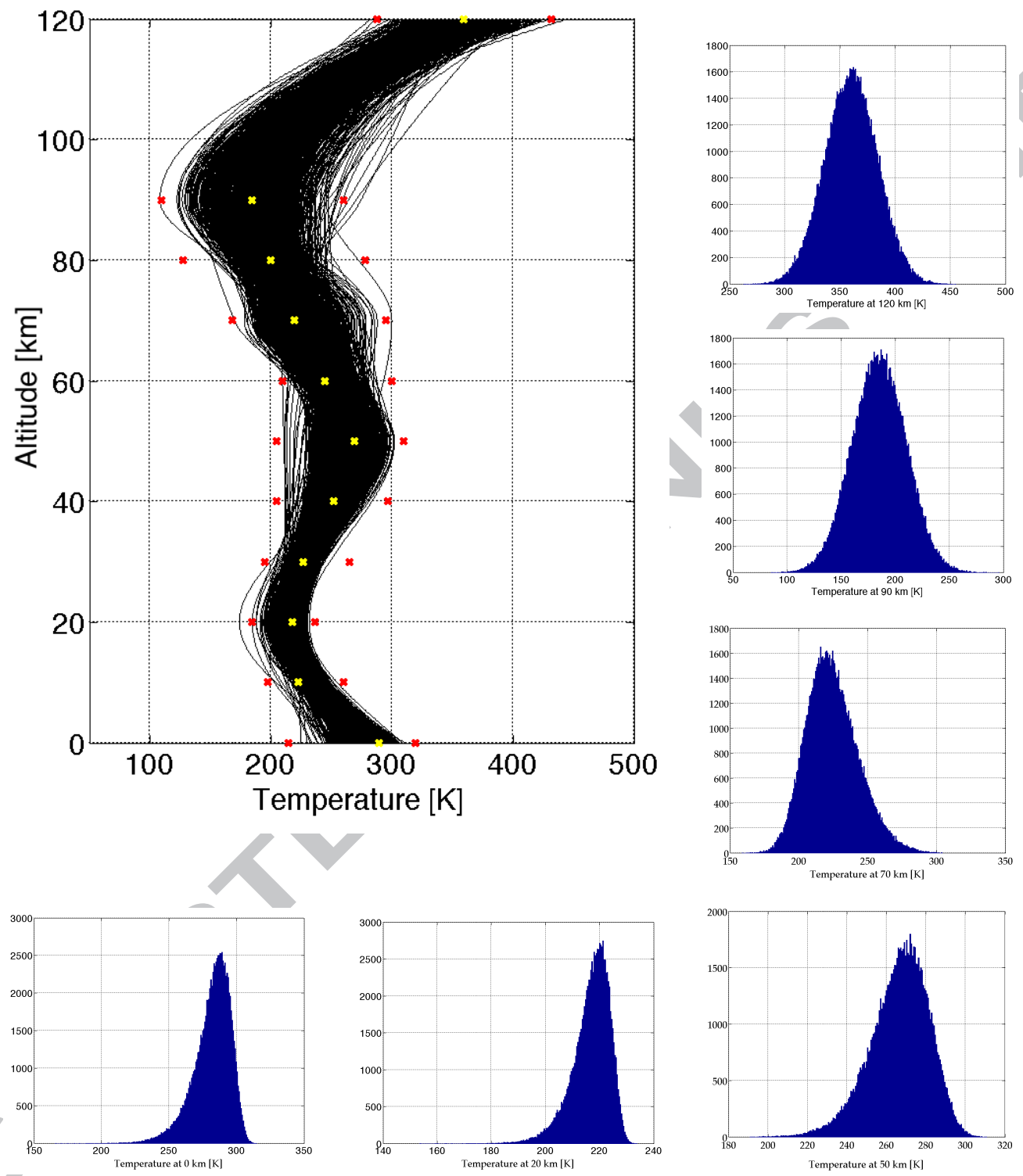

Figure 6: 1000 samples from the temperature interpolation routine along with the distribution histograms of the temperature stochastic input variables. The yellow and red data points represent the mean and boundaries for $99 \%$ of all samples, respectively. US Standard Atmosphere (1976) 

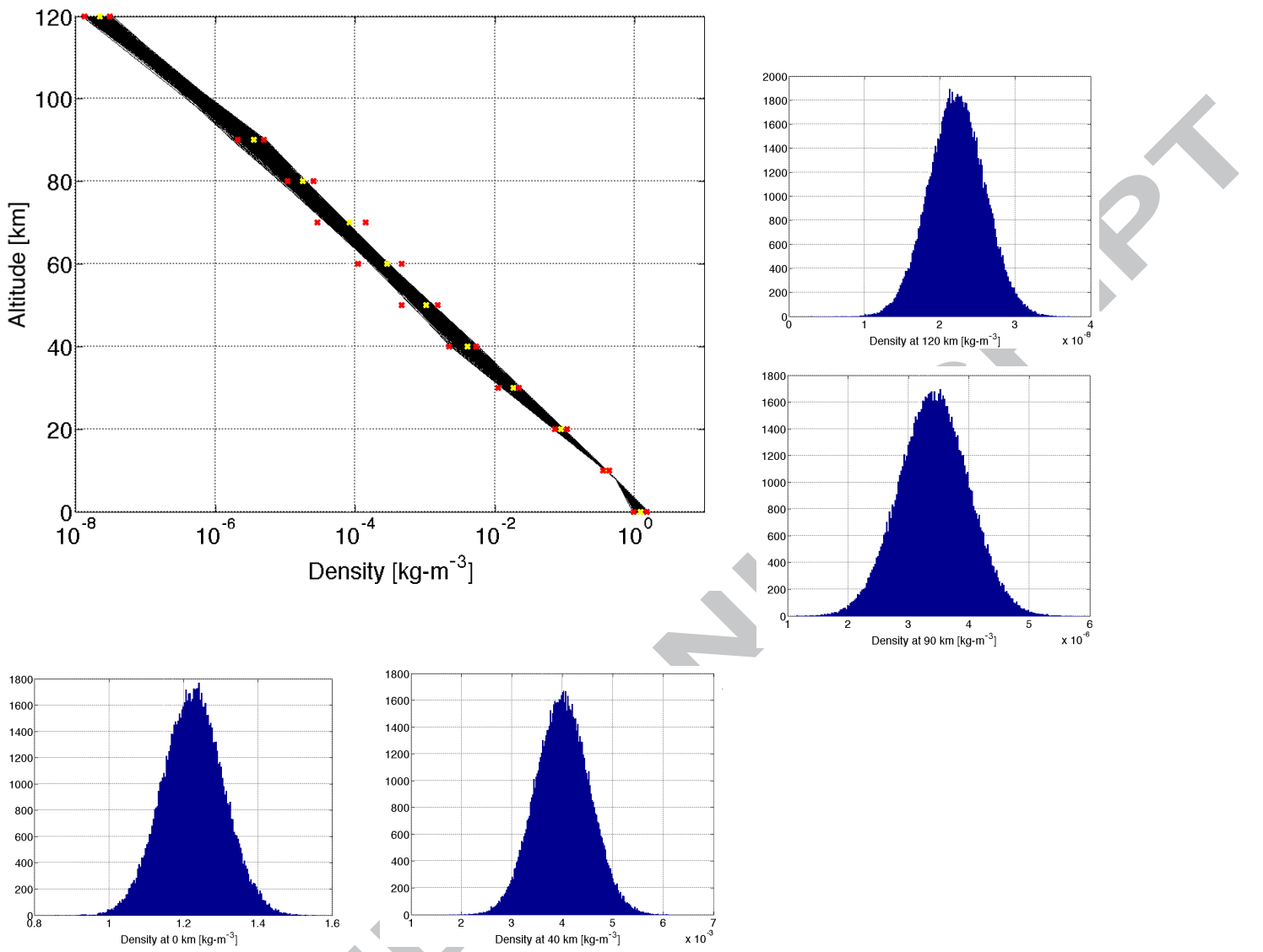

Figure 7: 1000 samples from the density interpolation routine along with the distribution histograms of the density stochastic input variables. The yellow and red data points represent the mean and boundaries for $99 \%$ of all samples, respectively. US Standard Atmosphere (1976) 


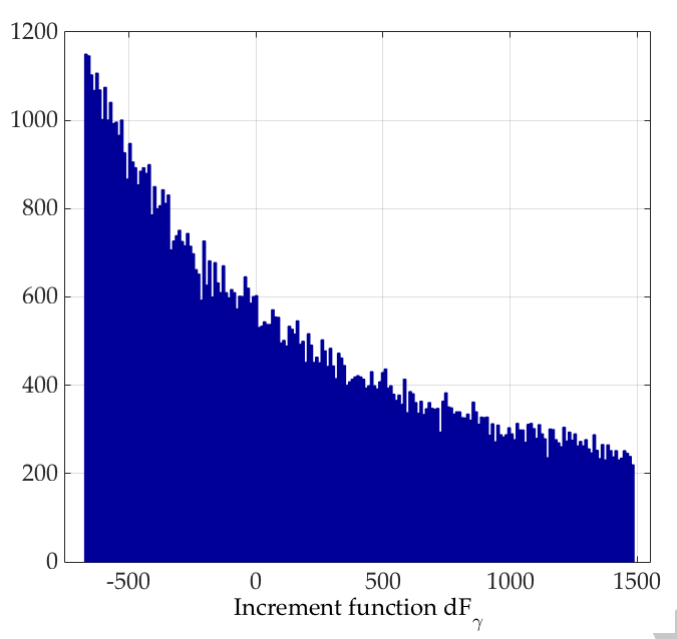

(a)

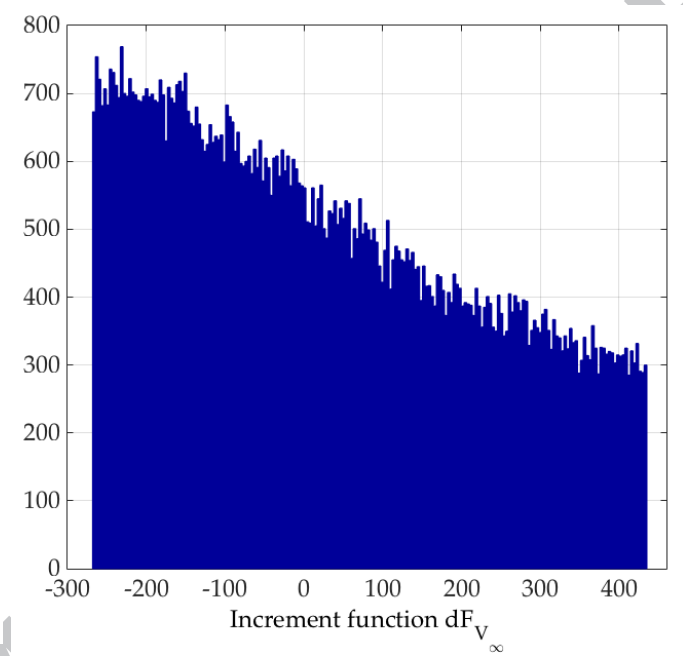

(b)

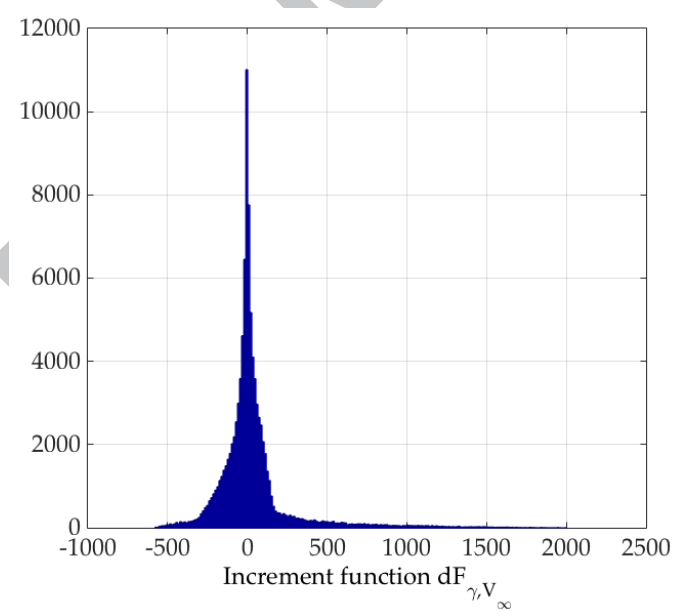

(c)

Figure 8: Partial histograms of the increment functions for the longitudinal impact distance of the 16D controlled 'normal' case. 


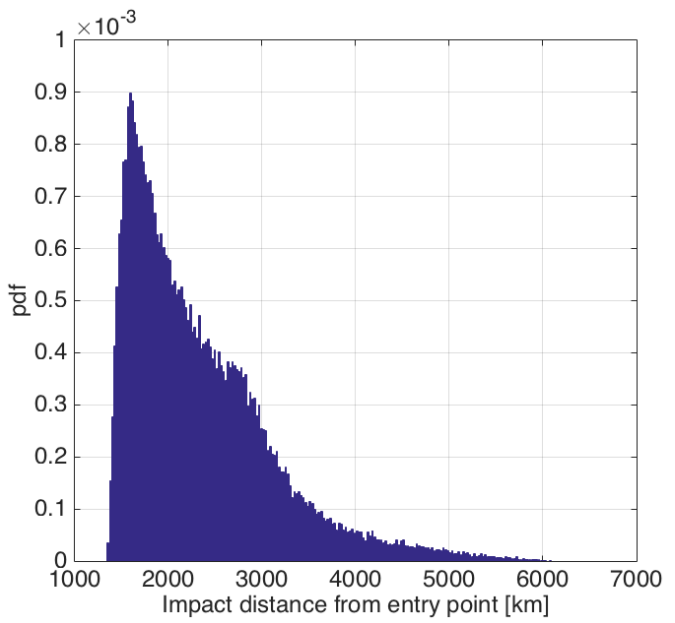

(a)

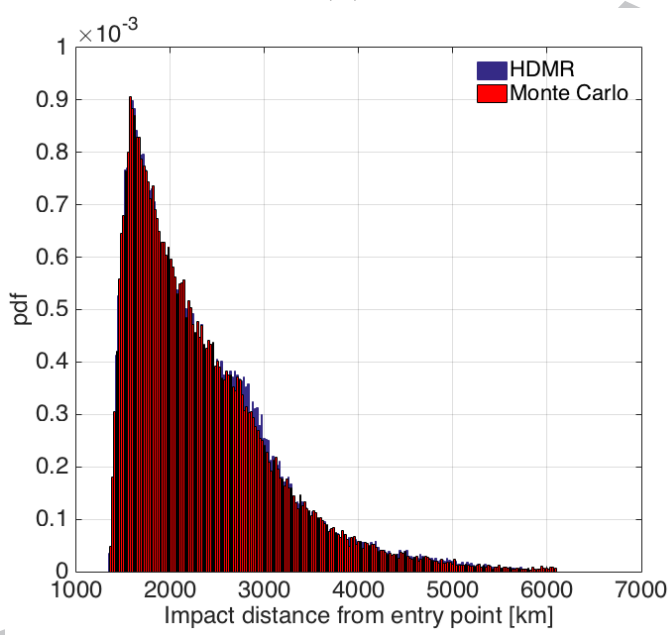

(c)

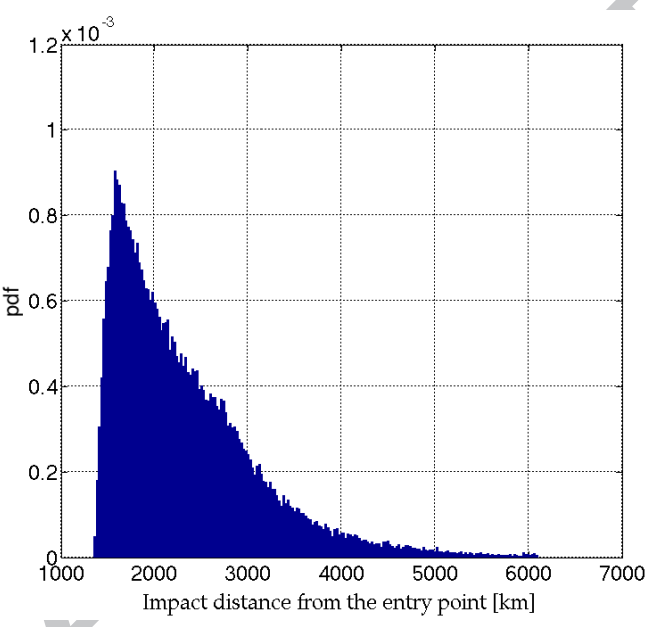

(b)

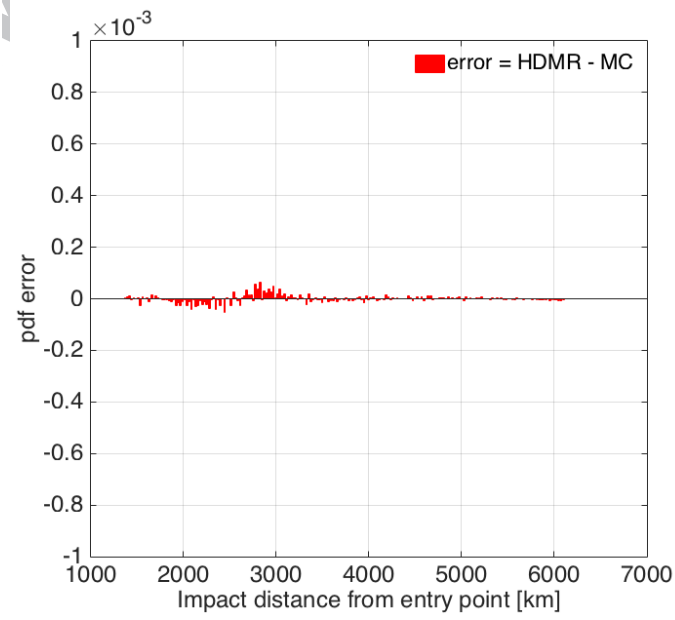

(d)

Figure 9: Probabilistic distribution for the 16D controlled 'normal' case in the longitudinal direction using (a) HDMR, and (b) Monte Carlo. (c) Overlay of the HDMR and MC histograms. (d) PDF error histogram. 


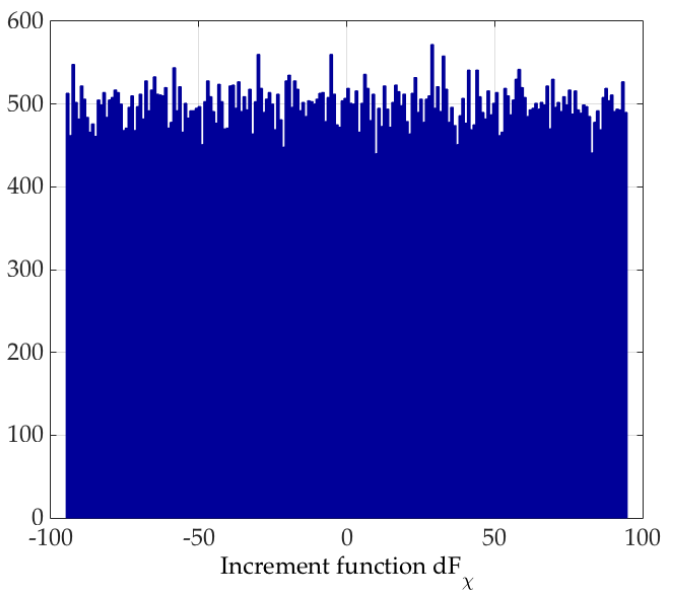

(a)

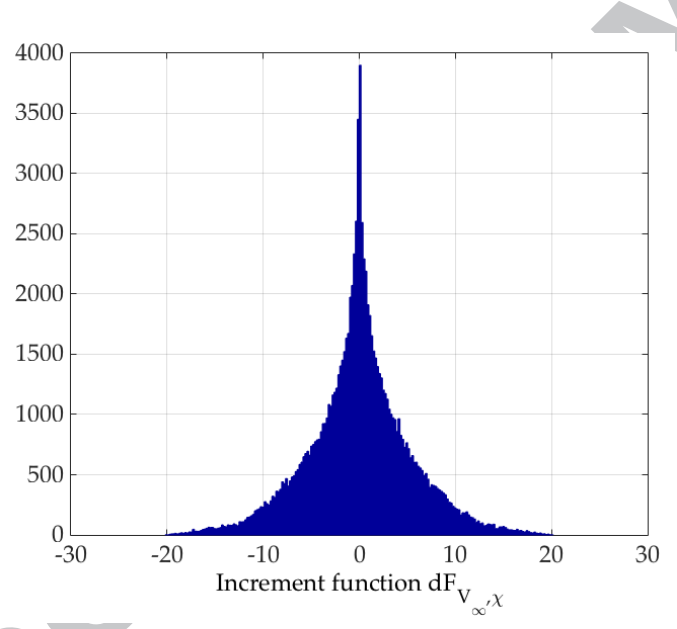

(c)

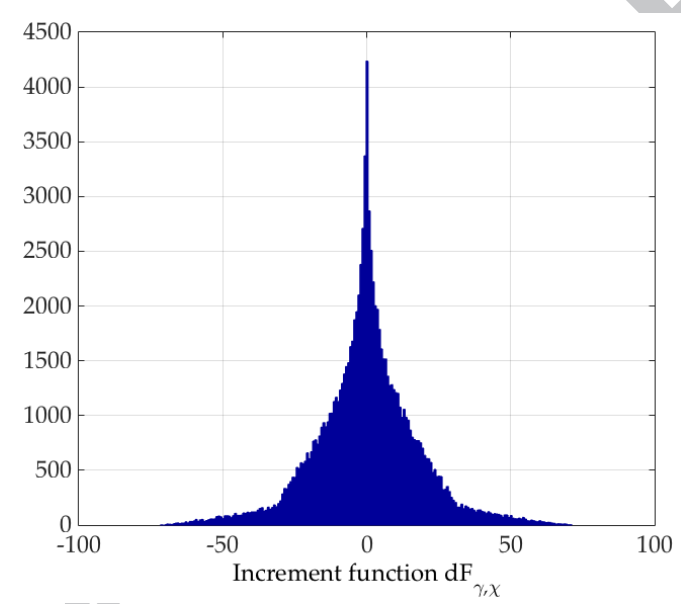

(b)

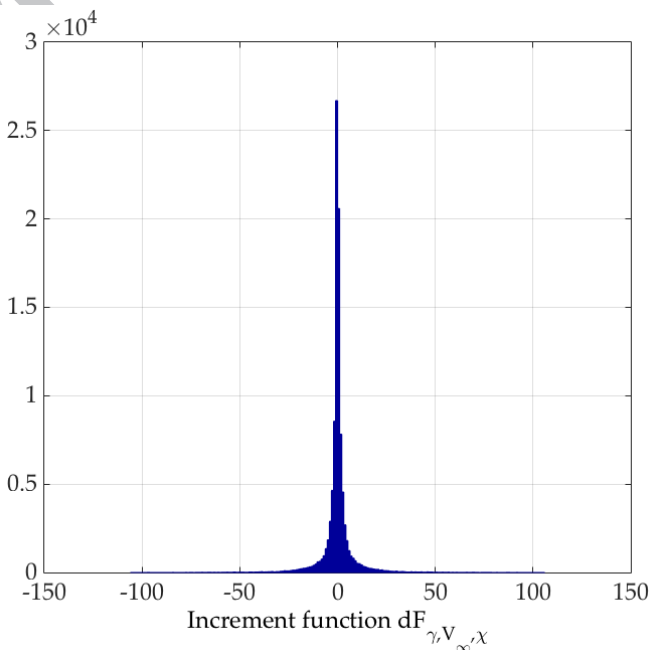

(d)

Figure 10: Partial histograms of the increment functions for the lateral spreadiness of the $16 \mathrm{D}$ controlled 'normal' case. 


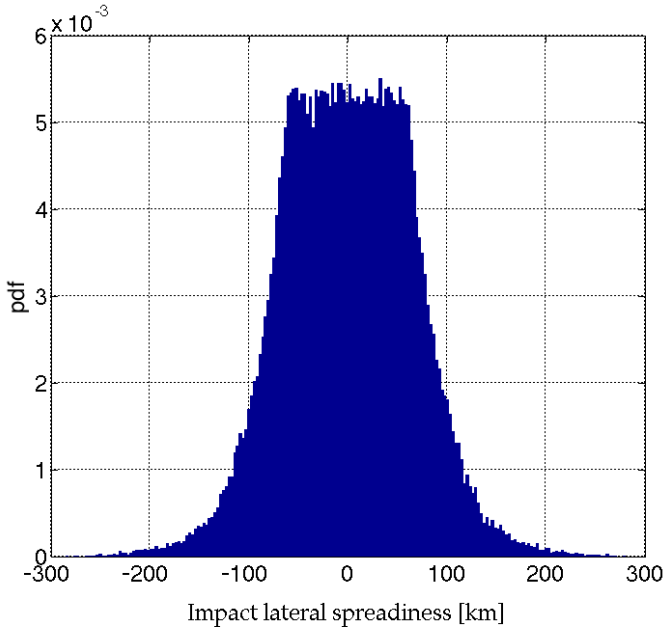

(a)

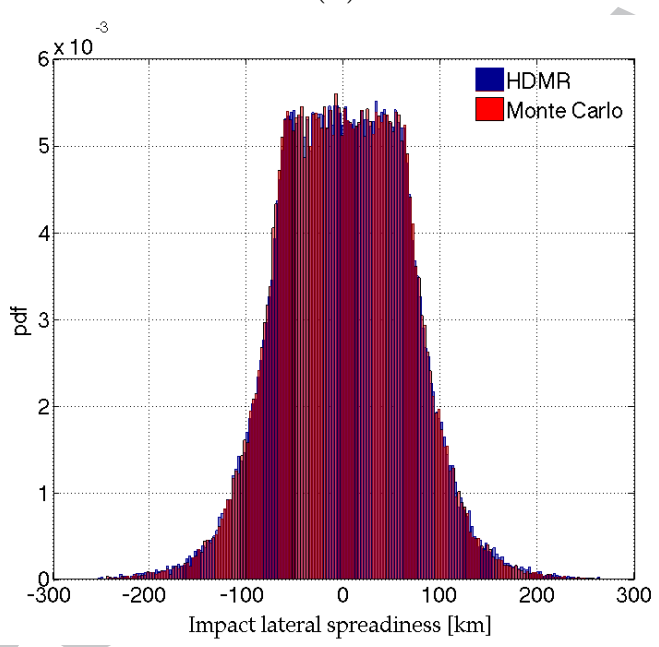

(c)

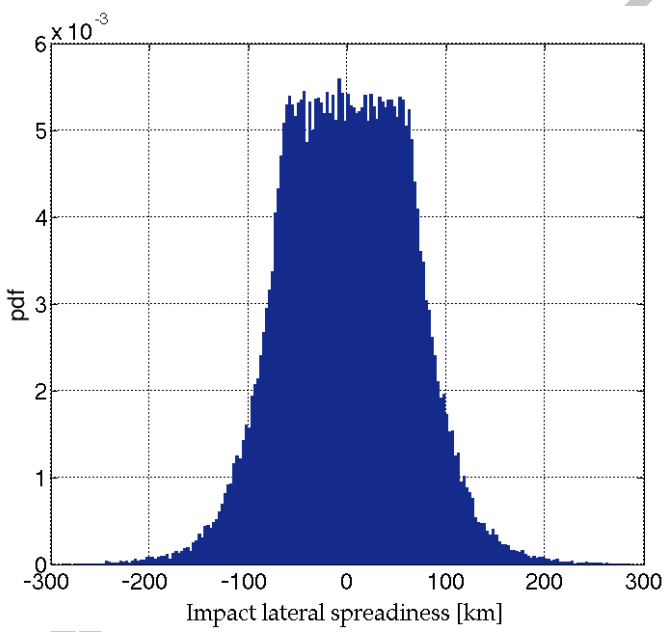

(b)

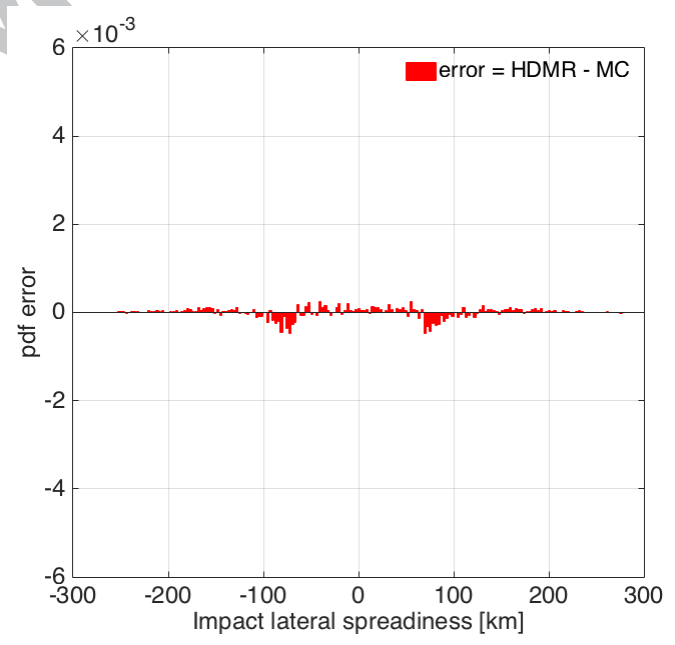

(d)

Figure 11: Probabilistic distribution for the 16D controlled 'normal' case in the lateral direction using (a) HDMR, and (b) Monte Carlo. (c) Overlay of the HDMR and MC histograms. (d) PDF error histogram. 


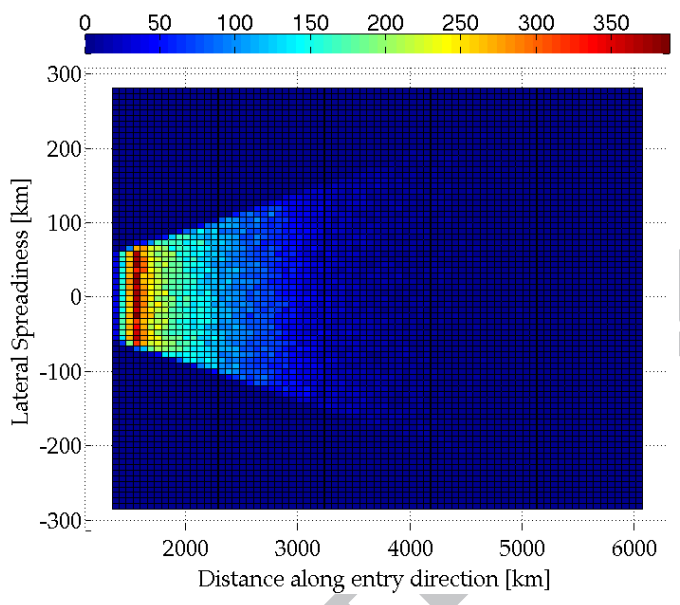

(a)

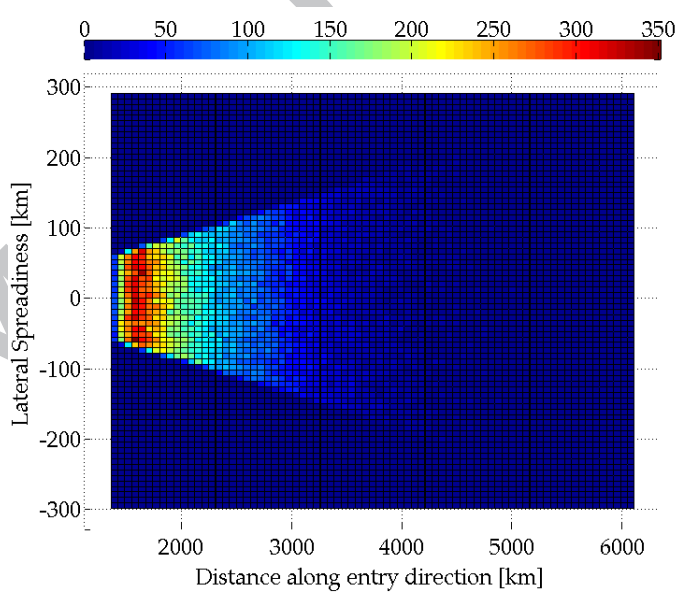

(b)

Figure 12: 2D histogram of the impact location for the 16D controlled 'normal' case using (a) HDMR, and (b) Monte Carlo. 


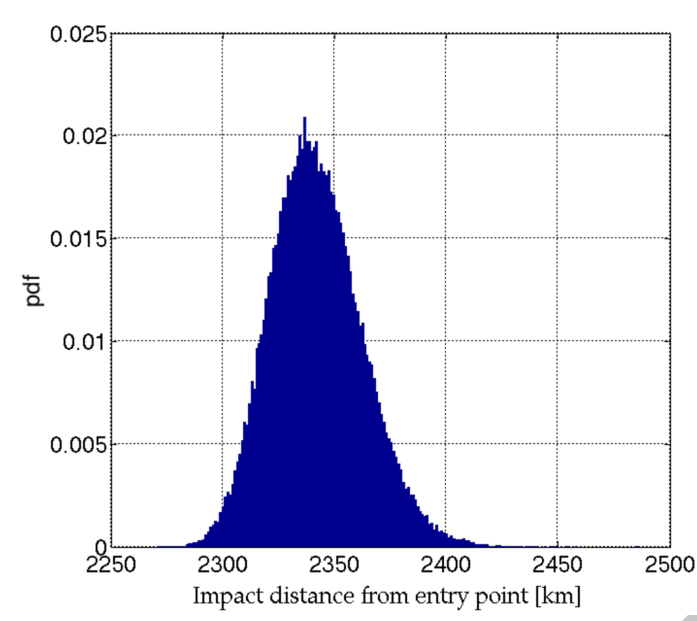

(a)

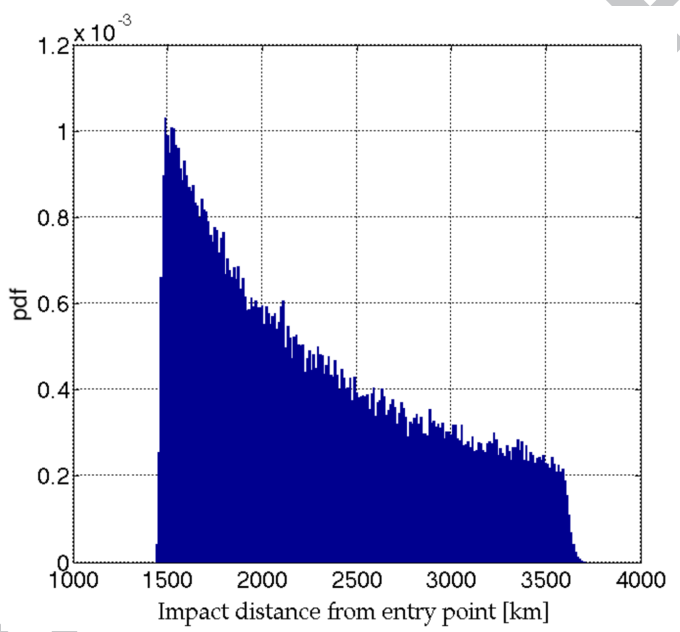

(b)

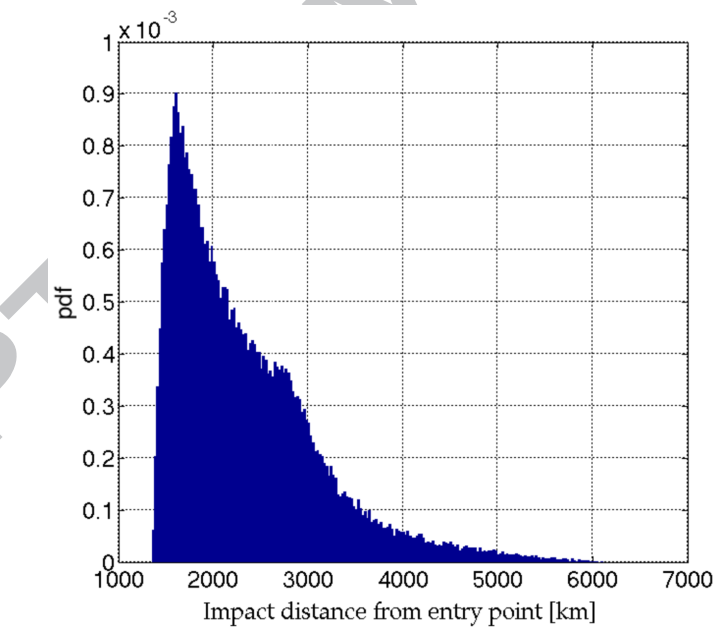

(c)

Figure 13: Probabilistic distribution of the longitudinal impact distance for controlled 'normal' (a) the 12D case, (b) the 13D case, and (c) the 15D case. 


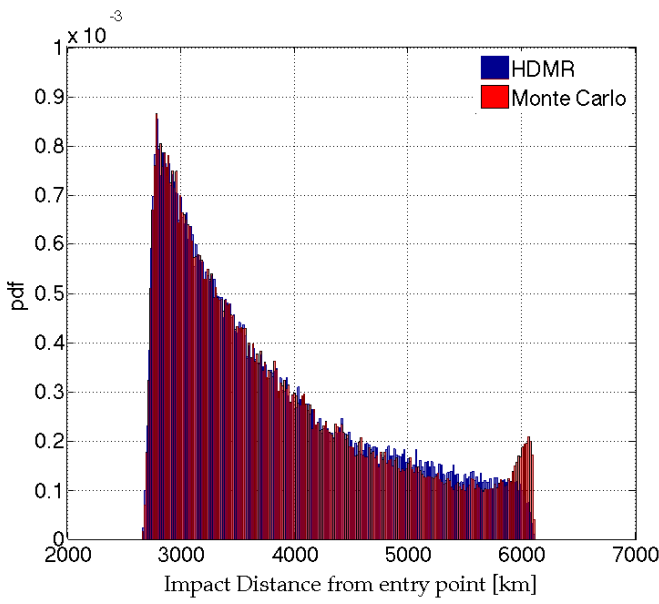

(a)

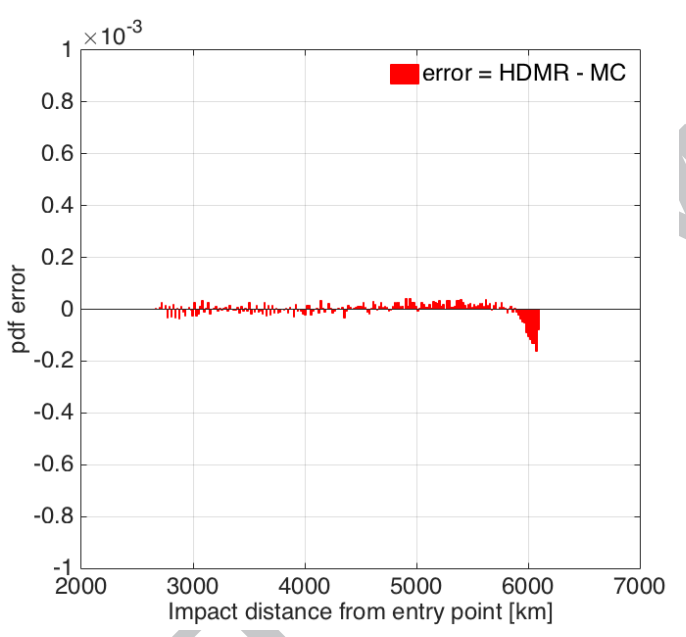

(c)

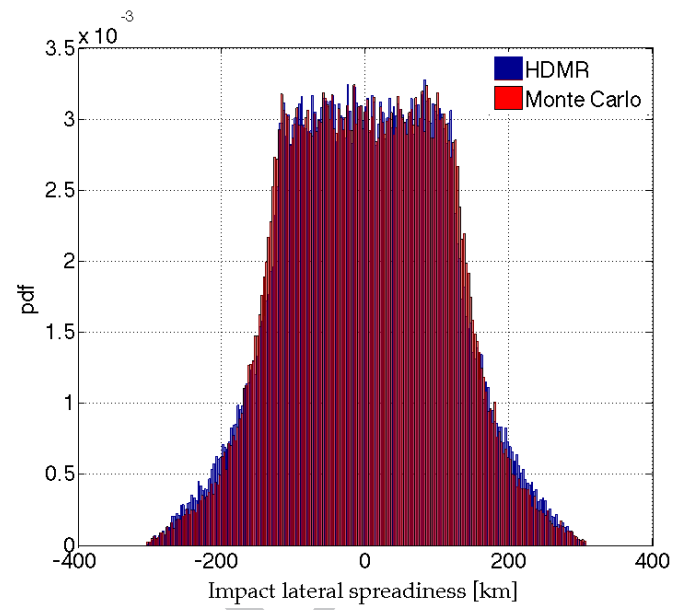

(b)

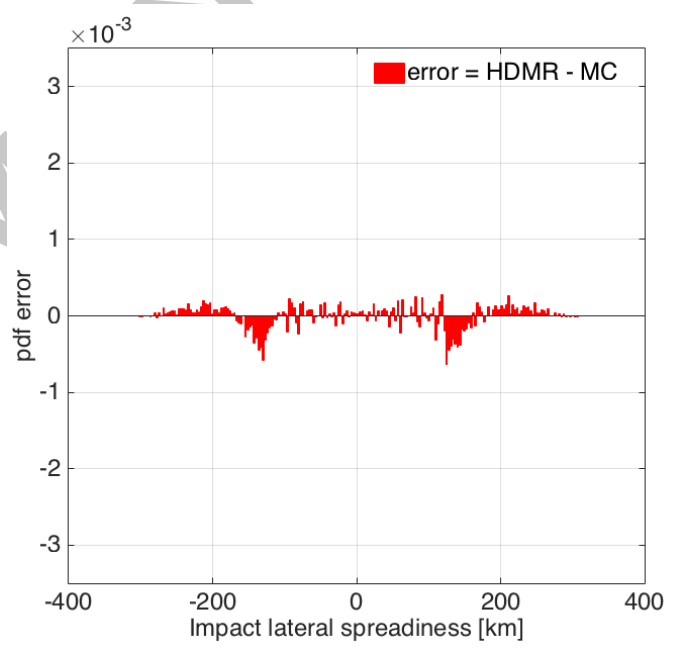

(d)

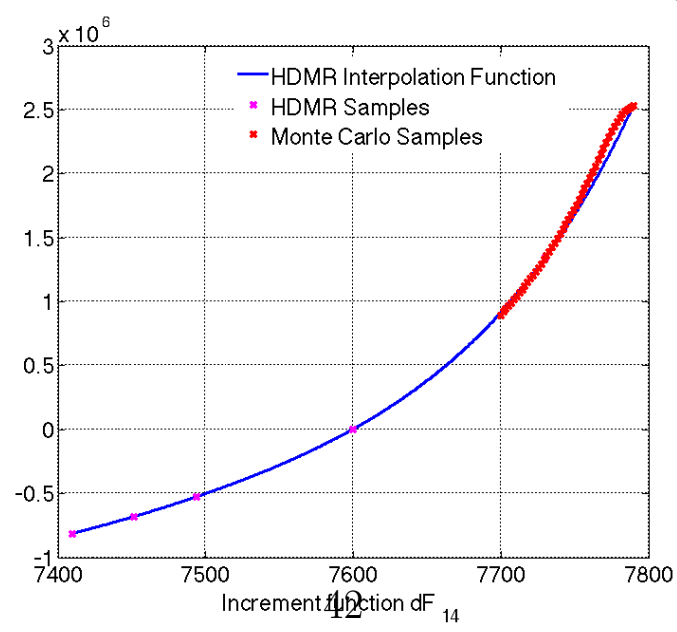

(e)

Figure 14: Overlay of the HDMR and MC histograms for un-controlled 'shallow' re-entry in the (a) longitudinal and (b) lateral directions. Error histograms in (c) longitudinal and (d) lateral directions. (e) The cause of the peak at the tail of the longitudinal HDMR pdf. 


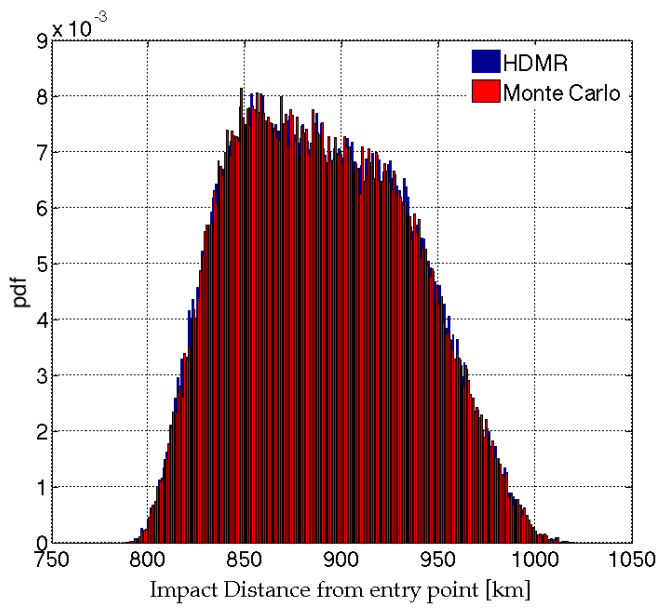

(a)

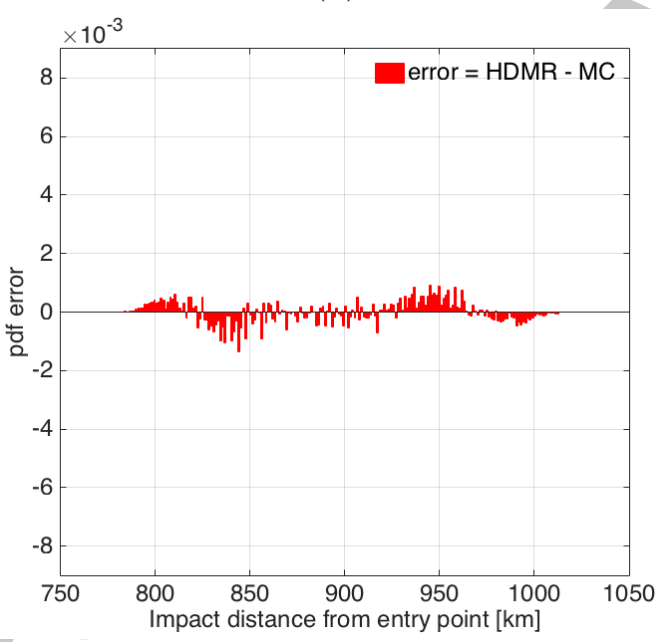

(c)

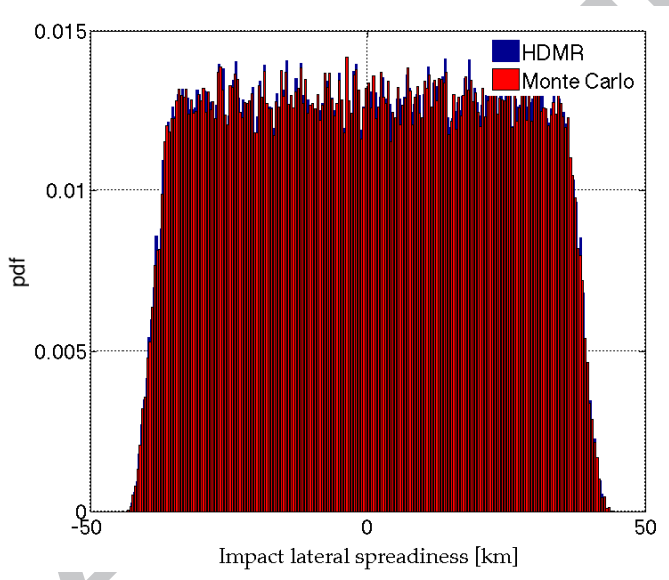

(b)

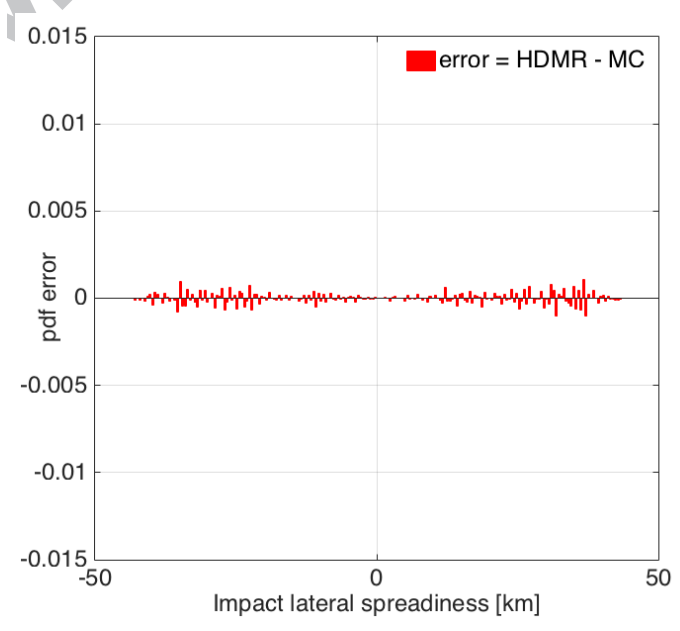

(d)

Figure 15: Overlay of the HDMR and MC histograms for controlled 'steep' re-entry. Error histograms in (c) longitudinal and (d) lateral directions. 